\title{
Philosophiques
}

\section{Index 1974-2004}

\section{Frédéric Tremblay}

Volume 31, numéro 2, automne 2004

URI : https://id.erudit.org/iderudit/009828ar

DOI : https://doi.org/10.7202/009828ar

Aller au sommaire du numéro

Éditeur(s)

Société de philosophie du Québec

ISSN

0316-2923 (imprimé)

1492-1391 (numérique)

Découvrir la revue

Citer ce document

Tremblay, F. (2004). Index 1974-2004. Philosophiques, 31(2), 461-506.

https://doi.org/10.7202/009828ar

Ce document est protégé par la loi sur le droit d'auteur. L'utilisation des services d'Érudit (y compris la reproduction) est assujettie à sa politique d'utilisation que vous pouvez consulter en ligne.

https://apropos.erudit.org/fr/usagers/politique-dutilisation/
Cet article est diffusé et préservé par Érudit.

Érudit est un consortium interuniversitaire sans but lucratif composé de l’Université de Montréal, l'Université Laval et l'Université du Québec à Montréal. Il a pour mission la promotion et la valorisation de la recherche. https://www.erudit.org/fr/ 


\section{Numéros thématiques}

Poincaré et la théorie de la connaissance, sous la direction de Éric Audureau, vol. 31, nº 1, p. 3-212.

Bernard Bolzano: Philosophie de la logique et théorie de la connaissance, sous la direction de Sandra Lapointe, vol. 30, $\mathrm{n}^{\circ}$ 1, p. 1-243.

La démocratie délibérative, sous la direction de Dominique Leydet, vol. 29, n² 2, p. 175-370.

Spinoza sous le prisme de son anthropologie, sous la direction de Syliane Charles et Jacques Henri Gagnon, vol. 29, n 1, p. 1-146.

La nature des normes, sous la direction de Christine Tappolet et Daniel Weinstock, vol. 28, $\mathrm{n}^{\circ} 1$, p. 3-203.

Le matérialisme contemporain, sous la direction de Paul Bernier, vol. 27, n 1, p. 3-186.

La critique de la raison en Europe centrale, sous la direction de Jean-Pierre Cometti et Kevin Mulligan. Actes du Colloque de Cerisy, vol. 26, n 2, p. 175-371.

Les modèles d'évolution en économie et en sciences sociales, sous la direction de Jean Mathiot et Robert Nadeau, vol. 25, n², p. 151-279.

Avez-vous lu Rawls?, sous la direction de Bjarne Melkevik, vol. 24, nº 1, p. 3-89.

Critères esthétiques et métamorphoses du beau, sous la direction de Suzanne Foisy, vol. 23, n 1 , p. 5-123.

Les femmes et la société nouvelle, sous la direction de Guy Bouchard, vol. 21, n² 2, p. 299-501. Perspectives sur la phénoménologie et l'intentionnalité, sous la direction de Denis Fisette, vol. 20, $\mathrm{n}^{\circ} 2$, p. 247-472.

Une nation peut-elle se donner la Constitution de son choix?, sous la direction de Michel Seymour, vol. 19, n², p. 5-214.

Égalité, justice et différence, sous la direction de Louise Marcil-Lacoste, vol. 11, n 2, p. 113-413. Le marxisme cent ans après Marx, vol. 10, $\mathrm{n}^{\circ}$ 2, p. 205-340.

\section{Articles}

ALLARD, Gérard. "Au-delà et en deçà de l'homme social : les pôles de la pensée de Rousseau ». Vol. 14, n² 2, p. 263-298.

ALLARD, Guy-H. «L'énigme et la culture littéraire d'Augustin ». Vol. 1, n² 2, p. 61-78.

AMSELEK, Paul. «La science et le problème de la liberté humaine ». Vol. 27, n² 2, p. 403-423. 
ATLAN, Henri. «Projet et signification dans des réseaux d'automates : le rôle de la sophistication ». Vol. 20, n² 2, p. 443-472.

AUMÈTRE, Jacques. «Habermas et Althusser : critique de l'idéologie scientiste et critique de l'humanisme idéologique». Vol. 15, n 1, p. 141-167.

BAERTSCHI, Bernard. «La place du normatif en morale». Vol. 28, n 1, p. 69-86.

BALDWIN, Thomas. «La valeur intrinsèque chez Brentano et Moore». Vol. 26, n 2, p. 231-243.

BAUGH, Bruce. "Temps, durée et mort chez Spinoza». Vol. 29, n 1, p. 23-39. . «De l'individu à l'histoire : l'authenticité dans les écrits de Sartre». Vol. 18, n² 2, p. 101-122.

BEAUSOLEIL, Jocelyn R. «À quoi peut servir la notion d'égalité ?» Vol. 11, n 1, p. 137-155.

BÉLANGER, Jean. «Images et réalités du behaviorisme». Vol. 5, n¹, p. 3-110.

BELLEFLEUR, Michel. "Travail et loisir. Du loisir antique au loisir contemporain». Vol. 8, $\mathrm{n}^{\circ} 2$, p. 303-341.

BELLEMARE, Pierre. «Symboles : fondements anthropobiologiques de la doctrine aristotélicienne du langage ». Vol. 9 , n 2, p. 265-279.

BENOIST, Jocelyn. «Propriété et détermination : Sémantique et ontologie chez Bernard Bolzano ". Vol. 30, n 1, p. 137-148.

BERNIER, Paul. «Introduction». Vol. 27, n 1, p. 3-10.

. «Fonctionnalisme et similarité phénoménale». Vol. 27, n 1, p. 99-114.

. " "Suivre une règle" chez Wittgenstein : un paradoxe sceptique pour Saul Kripke». Vol. 15, n² 2, p. 389-404.

BERSIANIK, Louky. «L'utopie de la gynilité, un non-lieu provisoire». Vol. 21, n² 2, p. 459-469.

BILODEAU, Renée. "La satisfaction d'être dupe ». Vol. 28, n 2, p. 381-394.

BLAIS, François. "Avortement, éthique sociale et positivisme juridique». Vol. 18, n 2, p. 63-78. . «Réformisme pénal et responsabilité : une étude philosophique». Vol. 16, n² 2, p. 293-325.

BLAIS, François, et Marcel FILION. "De l'éthique environnementale à l'écologie politique. Apories et limites de l'éthique environnementale». Vol. 28, n² 2, p. 255-280.

BONIFACE, Jacqueline. «Poincaré et le principe d'induction». Vol. 31, n 1, p. 131-149.

BONIN, Pierre-Yves. «Les deux libéralismes de Charles Taylor : le Québec et le Canada ». Vol. 22, $\mathrm{n}^{\circ} 1$, p. 3-20.

BORDELEAU, Léo-Paul. «Un nouveau paradigme : le corps sportif» (1). Vol. 12, n 1, p. 33-51. . «Un nouveau paradigme : le corps sportif» (2). Vol. 12, n² 2, p. 247-279. . «Une genèse de la vie sociale selon Maurice Blondel ». Vol. 2, n 1, p. 55-82.

BOUCHARD, Guy. "Les modèles féministes de société nouvelle ». Vol. 21, n 2, p. 483-501. . «Typologie des tendances théoriques du féminisme contemporain». Vol. 18, n 1, p. 119-167.

. «La métaphore heuristique de l'esclavage dans les textes féministes ». Vol. 14, n 1 , p. 121-144.

. "Marx, Bloch et l'idéologie». Vol. 10, n², p. 265-288.

. «Gilson, l'œuvre d'art et le roman». Vol. 9, n² 2, p. 195-221.

. «Le recours à l'auditoire universel implique-t-il une pétition de principe ? ". Vol. 7, n² p. 161-188. 
BOUCHARD, Guy, et Maria DE KONINCK. «Introduction». Vol. 21, n² 2, p. 299-302.

BOUCHARD, Roch. «L'idéalisme est-il spiritualiste?» Vol. 12, n 1, p. 53-71.

BOULAD-AYOUB, Josiane. «Les écrits politiques de d'Holbach et la Déclaration des Droits de $1789 »$. Vol. 18, n 2, p. 123-137.

. «"Et la religion le remplit de fureur...”. Les déterminations idéologiques, polémiques et politiques du Mahomet de Voltaire». Vol. 17, n² 2, p. 3-22.

. " "Il faut être idéologiste pour seulement en avoir l'idée...”. Quelques remarques à propos du chapitre V du tome II des Éléments d'idéologie de D. de Tracy». Vol. 13, n 1, p. 39-52.

. «Éros androgyne et logos philosophique». Vol. 12, n 1, p. 107-132.

. «Descartes face à Leibniz sur la question de la substance». Vol. 11, n 2, p. 225-249.

. «L'image du centre et la notion de l’Un dans les Ennéades». Vol. 11, n 1, p. 41-70.

. «La leçon de l’idéologie allemande». Vol. 10, n² 2, p. 221-241.

BOURDEAU, Michel. «Grammaire et psychologie. Remarques sur la nature et les origines de la grammaire générative». Vol. 10, n 1, p. 75-96.

BOURNEUF, Éric. «Pour une gnoséologie objective et applicable». Vol. 23, n 2, p. 265-283. . «Référence et dénotation des termes scientifiques». Vol. 18, n² 2, p. 27-62.

BOVE, Laurent. «De l'étude de l'État hébreu à la démocratie : La stratégie politique du conatus spinoziste». Vol. 29, n 1, p. 107-119.

BRANDOM, Robert B. «Quelques thèmes pragmatistes dans l'idéalisme de Hegel ». Vol. 27, $\mathrm{n}^{\circ} 2$, p. 231-261.

BRENNER, Anastasios. «Géométrie et genèse de l'espace selon Poincaré ». Vol. 31, n 1, p. 115-130.

BRIEF, Jean-Claude. «Le constructivisme piagétien et les épistémologies traditionnelles ». Vol. 4, $\mathrm{n}^{\circ} 2$, p. 195-224.

BRODEUR, Jean-Paul. «De l'orthodoxie en philosophie. À propos de l'Académie canadienne SaintThomas d'Aquin». Vol. 3, n² 2, p. 209-253.

. "Justice distributive et justice rétributive». Vol. 24, n 1, p. 71-89.

BROSSARD, Nicole. «Écrire la société : d'une dérive à la limite du réel et du fictif». Vol. 21, n 2 , p. 303-320.

BROUSSON-ROSSAY, Marie-Christine. «Psychanalyse et féminité». Vol. 12, n 1, p. 177-190.

BUCHANEN, Allen. «Les conditions de la sécession ». Vol. 19, n 2, p. 159-168.

BUCKLEY, Philip R. «La notion d'authenticité chez Husserl et Heidegger». Vol. 20, n² 2, p. 399-422.

CALDWELL, Gary. "Le Québec ne doit pas se donner une Constitution : il en a déjà une qu'il abandonnerait à ses risques et périls». Vol. 19, n² 2, p. 191-198.

CALLAN, Eamonn. « Réconciliation et éthique de la mémoire publique». Vol. 29, n 2, p. 311-326.

CANTIN, Serge. "L'homme de Marx est-il un sujet individuel ou un être social ? À propos de l'interprétation de Marx par Louis Dumont». Vol. 18, n 1, p. 25-60.

. «De l'illusion à l'espérance». Vol. 15, n² 2, p. 251-310.

CARIGNAN, Maurice. "L'éternel comme tiers synthétisant chez Kierkegaard». Vol. 8, n 1 , p. 75-92. 


\section{Philosophiques / Automne 2004}

CARON, Jacques. «Dialectique de la communication chez Kierkegaard». Vol. 3, n 2, p. 167-181.

CAYLA, Fabien. «Husserl, Brentano et la psychologie descriptive». Vol. 20, n 2, p. 347-361.

CHARETTE, Léon. «Droit naturel et droit positif chez saint Thomas d'Aquin ». Vol. 8, $\mathrm{n}^{\circ} 1$, p. $113-130$.

CHARLES, Daniel. «Pour une esthétique sans privilèges ». Vol. 23, n 1, p. 125-130.

CHARLES, Syliane. «Le salut par les affects : la joie comme ressort du progrès éthique chez Spinoza ». Vol. 29, n 1, p. 73-87.

CHARLES, Syliane, et Jacques-Henri Gagnon. «Introduction». Vol. 29, n 1, p. 3-5.

. «Réconciliation et dépassement de l'art par la philosophie chez Hegel : une analyse critique». Vol. 25, n 1, p. 49-61.

CHARRON, Ghyslain. «Inconscient social de la psychanalyse et points aveugles du psychanalyste». Vol. 4, n² 2, p. 293-304.

CLAVET, Jean-Claude. «Le concept de liberté chez Herbert Marcuse». Vol. 13, n² 2, p. 209-235.

CLAYBOROUGH, Arthur. «La grammaticalité des textes littéraires». Vol. 8, n 1, p. 93-111.

CLOUTIER, Yvan. "Par delà le féminisme : pour une éthique sartrienne de la pluralité des points de vue». Vol. 21, $\mathrm{n}^{\circ}$ 2, p. 333-341.

. «Philosophie et marketing : Sartre à Montréal, mars 1946 ». Vol. 15, n 1, p. 169-190.

. «Gramsci et la question de l'idéologie». Vol. 10, n² 2, p. 243-253.

CORNETTI, Jean-Pierre, et Kevin MULLIGAN. «Avant-Propos». Vol. 26, n² 2, p. 175.

. «Raison, argumentation et légitimation. Habermas, Apel et les apories de la communication ». Vol. $19, n^{\circ} 1$, p. 3-24.

CORNU, Roger. «Évolution et processus configurationnel chez Norbert Elias ». Vol. 25, n 2 , p. 239-256.

CÔTÉ, Jean-Guy. «Apport féminin et champ de la psychanalyse». Vol. 21, n 2, p. 405-416.

COUTINE, Jean-Jacques. «Définition d'orientations théoriques et construction de procédures en analyse du discours ». Vol. 9, n² 2, p. 239-264.

COURTOIS, Stéphane. «Habermas et la question du nationalisme : le cas du Québec ». Vol. 27, $\mathrm{n}^{\circ} 2$, p. 377-401.

COUTEL, Charles. "De Thomas Moore à Condorcet : une relève du discours utopique? » Vol. 24, n² 2, p. 277-284.

COUTURE, Jocelyne. «Explication et justification en philosophie morale». Vol. 28, n 1, p. 129-150.

. «L'institutionnalisation de la raison publique : le moral et le politique». Vol. 24, $\mathrm{n}^{\circ} 1$, p. 43-57.

. «L'art de la séparation ». Vol. 19, n², p. 41-54.

CROCCO, Gabriella. «Intuition, construction et convention dans la théorie de la connaissance de Poincaré». Vol. 31, n 1, p. 151-177.

DAVION, Victoria. «Souci et connexion dans l'éthique de la politique générale ». Vol. 22, n 1 , p. 53-63.

DE CUZZANI, Paola. "Une anthropologie de l'homme décentré ». Vol. 29, n 1, p. 7-21.

DE KONINCK, Maria et Guy BOUCHARD. «Introduction». Vol. 21, n 2, p. 299-302. 
DEVILLAIRS, Laurence. "La Forme méditative de la métaphysique cartésienne ». Vol. 28, n², p. 281-302.

DIOUSSOU, Arianne Michelle. «Femmes, hommes et féministes à la barre». Vol. 21, n 2, p. 343-355.

DOKIC, Jérôme. "Qui a peur des qualia corporels ?" Vol. 27, n 1, p. 77-98.

DRAY, William H. «Les explications causales en histoire». Vol. 4, n 1, p. 3-34.

DREYFUS, Hubert. «Agir, intentionnalité et être-au-monde ». Vol. 20, n 2, p. 285-302.

DUCHESNAU, François. «Leibnitz et les hypothèses de physique ». Vol. 9, n 2, p. 223-238. . "Sémiotique et abstraction : de Locke à Condillac". Vol. 3, n² 2, p. 147-166.

DUBUCS, Jacques et Sandra Lapointe. «Preuves par excellence». Vol. 30, n 1, p. 219-234.

DUFOUR, Christian. «La société distincte ». Vol. 19, n² 2, p. 55-61.

DUMOUCHEL, Daniel. «La dialectique du beau et du sublime : l'héritage kantien d'Adorno ». Vol. 23, n 1, p. 37-46.

ENGEL, Pascal. «Le "patternalisme” de Dennett». Vol. 22, n² 2, p. 197-212.

ERTLER, Klaus-Dieter. "La théorie des systèmes luhmanienne : un nouveau paradigme en sciences sociales?». Vol. 21, $n^{\circ} 1$, p. 3-17.

EVERETT, James, et Alain Morin. "Conscience de soi et langage intérieur : quelques spéculations ». Vol. 17, n² 2, p. 169-188.

FAUCHER, Luc. «Émotions fortes et constructionnisme faible». Vol. 26, n 1, p. 3-35.

FAURÉ, Christine. "Le statut du serment et de la promesse dans la Déclaration des Droits de $1789 »$. Vol. $19, n^{\circ} 1$, p. $75-85$

FILION, Marcel, et François BLAIS. "De l'éthique environnementale à l'écologie politique. Apories et limites de l'éthique environnementale». Vol. 28, n² 2, p. 255-280.

FISETTE, Denis. «Les suites de la phénoménologie». Vol. 20, n² 2, p. 247-265.

. «Les cours de Marbourg et la phénoménologie». Vol. 20, n² 2, p. 303-321.

. «Pensée, langage et perception». Vol. 18, n² 2, p. 79-100.

FOISY, Suzanne. «Présentation». Vol. 23, n 1, p. 5-11. . «Différence esthétique et argumentation ». Vol. 23, n 1, p. 113-123.

FØLLESDAL, Dagfinn. «Le rôle de l'action dans la constitution du monde chez Husserl et Heidegger ». Vol. 20, n² 2, p. 267-284.

FRÉCHETTE, Guillaume. «Daubert et les limites de la phénoménologie : étude sur le donné et l'évidence». Vol. 28, n² 2, p. 303-326.

GAGNÉ, Learry. «La délibération circonstancielle en théorie démocratique ». Vol. 29, n 2, p. 327-350.

GAGNON, Carolle. «Nouvelles sociétés, nouveaux mythes? ». Vol. 21, n² 2, p. 471-481.

GAGNON, Jacques-Henri. «Spinoza et le problème de l'akrasia : Un aspect négligé de l'ordo geometricus». Vol. 29, n 1, p. 57-71.

GAGNON, Jacques-Henri, et Syliane Charles. «Introduction». Vol. 29, n 1, p. 3-5.

GAGNON, Maurice. «La critique piagétienne de l'apriorisme attaque-t-elle le criticisme kantien ?".Vol. 7, n 1, p. 41-54. 
. Présentation de «Essai de métaphysique» (Notes et jalons)» de Jean Goulet. Texte publié en hommage à Jean Goulet décédé en février 1979. Vol. 6, n² 2, p. 361-386.

. «Épistémologie génétique, science et philosophie». Vol. 4, n² 2, p. 225-244.

. «Une analyse sémantique du concept de causalité est-elle possible ?». Vol. 2, n² 2 , p. $187-205$

GALLUP, John. «L'amitié, la société et la femme». Vol. 21, n² 2, p. 417-431.

GARCEAU, Benoît. "Les travaux de jeunesse de Hegel et l'interprétation de sa philosophie de la religion ". Vol. 1, n 1, p. 21-49.

GARRETT, Brian. «Héritabilité causale et propriétés émergentes ». Vol. 27, n 1, p. 139-159.

GAUTHIER, Claude. «Spencer, le concept de société : entre organicisme et individualisme». Vol. 20, n 1 , p. 3-24.

GAUTHIER, Gilles. «L'engagement psychologique dans la communication langagière ». Vol. 16, $\mathrm{n}^{\circ} 1, \mathrm{p} .43-71$.

GAUTHIER, Yvon. «Notes sur la syntaxe et la sémantique du concept d'égalité ». Vol. 11, n², p. 349-352.

. "Vérité et vérification en logique mathématique et dans les théories physiques ». Vol. 9, $n^{\circ} 1$, p. 135-145.

. "Sur la théorie des démonstrations». Vol. 8, n² 2, p. 273-285.

GEORGE, Rolf. «Intuitions ». Vol. 30, n 1, p. 19-46.

GICQUEL, Hervé-Marie. «Le Livre sur Adler de Søren Kierkegaard». Vol. 12, n² 2, p. 315-362.

GIROUX, France. «De la société civile à l'État : l'irruption de l'inégalité». Vol. 11, n² 2, p. 373-387. - «Concept d'injustice sociale et absence d'intégration d'une classe en société civile». Vol. 10, n² 2, p. 361-375.

GIROUX, Laurent. «La connaissance historique via l'interliaison psychique : Wilhelm Dilthey ». Vol. 7, n² 2, p. 189-209.

. «Heidegger et la Métaphysique : vers un double dépassement». Vol. 2, n² 2, p. 207-228.

GODARD, Linda. «Pour une nouvelle lecture de la question de la "femme" : essai à partir de la pensée de Jacques Derrida». Vol. 12, n 1, p. 147-164.

GODIN, Christian. «Tensions et apories de l'encyclopédisme». Vol. 24, n² 2, p. 285-298.

GOETZ, Rose. «La place de l'éviction et de la fuite dans le perfectionnement éthique ». Vol. 29, $\mathrm{n}^{\circ} 1$, p. 89-98.

GOFFI, Jean-Yves. «La nouvelle casuistique et la naturalisation des normes ». Vol. 28, $\mathrm{n}^{\circ} 1$, p. $87-107$.

GOHIER, Christiane. «Le rapport masse-élite comme modèle canonique de la dialectique sociale. » Vol. 11, n 2, p. 337-348.

GOLDSTICK, Daniel. «Lecture d'Althusser». Vol. 12, n² 2, p. 363-391.

GOUIN, Jean-Luc. «La Raison comme lemme philosophique ou "Der Instinkt der Vernünftigkeit" - Pour accoster Hegel ». Vol. 23, n 2, p. 285-303.

. "Merleau-Ponty et le marxisme. Ou la difficulté de tolérer l'intolérable». Vol. 18, nº 1, p. 95-117. 
GOULET, Jean. «Essai de métaphysique». (Notes et jalons). Présenté par Maurice Gagnon. Texte publié par Maurice Gagnon en hommage à l'auteur décédé en février 1979. Vol. 6, $\mathrm{n}^{\circ} 2$, p. 361-386.

GRANGER, Gilles-Gaston. "Les conditions proto-logiques des langues naturelles ». Vol. 16, $n^{\circ} 2$, p. 245-256.

GRAVEL, Pierre. «Hegel et la construction de l'État. Contribution à sa destruction ». Vol. 9, n 1, p. 95-117.

. «Métaphore - Catharsis - Aufhebung ». Vol. 7, n² 2, p. 133-159.

. «Pour une logique de l'action tragique : Hegel et la tragédie». Vol. 5, n¹, p. 111-131.

GRIARD, Jérémie. «Le meilleur régime selon Leibniz». Vol. 31, n² 2, p. 349-372.

GRONDIN, Jean. «La contribution silencieuse de Husserl à l'herméneutique ». Vol. 20, n 2, p. 383-398.

GUAY, Éric. «Nature et esprit chez Hegel». Vol. 30, n² 2, p. 391-405.

. «Le savoir absolu hégélien, ou comment rentrer chez soi». Vol. 26, n 1, p. 71-82.

GUÉRIN, Michel. «La méthode du discours : la philosophie». Vol. 22, n 2, p. 247-263.

GUÉROULT, Martial. «Méthode en histoire de la philosophie». Vol. 1, n 1, p. 7-19.

GUERTIN, Marie. «L'orientation socialiste dans la philosophie politique de Shelley ». Vol. 6, $\mathrm{n}^{\circ} 2$, p. $253-271$

GUTMANN, Amy, et Dennis THOMPSON. «Pourquoi la démocratie délibérative est-elle différente?" Vol. 29, n² 2, p. 193-214.

HAACK, Susan. "Le bras long du sens commun. En guise de méthode scientifique ». Vol. 30, $\mathrm{n}^{\circ} 2$, p. 295-320.

HADREAS, Peter. «Searle versus Derrida ?». Vol. 23, n 2, p. 317-326.

HATZENBERGER, Antoine. "Réflexion complice et réflexion purifiante chez Sartre et Heidegger ». Vol. 25, n 1, p. 63-71.

HEATH, Joseph. «Brandom et les sources de la normativité ». Vol. 28, n 1, p. 27-46.

HENRY, Michel. «Qu'est-ce que nous appelons la vie?». Vol. 5, n 1, p. 133-150.

HJORT, Mette. «Le privilège culturel et la politique de la reconnaissance ». Vol. 23, n 1, p. 47-55.

HOUDE, Roland. "À propos ». (Réflexions). Vol. 5, n 1, p. 151-154.

HOULD, Richard L., et Marc-A. PROVOST. «Éthologie et cybernétique : leur approche à la psychologie». Vol. 7, n² 2, p. 301-319.

JACOB, Pierre. "Ce que pense un individu peut-il expliquer ce qu’il fait?». Vol. 27, n 1, p. 115-138. . «Fodor, la psychologie scientifique et les attributions de croyance». Vol. 20, n 1 , p. 131-158.

JACQUETTE, Dale. "Émergence et incorporation d'après Margolis ». Vol. 13, n 1, p. 53-63.

JOANNIS, David Guy. «Le projet épistémologique de Sartre». Vol. 23, n² 2, p. 305-315.

JOOS, Jean-Ernest. «Banalité du mal et sens du devoir chez les administrateurs de l'extermination». Vol. 19, n 1, p. 61-74.

KAHANE, David. «Délibération démocratique et ontologie sociale». Vol. 29, n² 2, p. 251-286. 
KAUFMANN, J. Nicolas. «Les trois niveaux de la preuve dans les sciences empirico-formelles ». Vol. $7, \mathrm{n}^{\circ} 2$, p. 247-265.

. "Contributions de l'école de Francfort à la "théorie" des idéologies». Vol. 5, n² 2, p. 229-250.

. «Psychologie de la "conscience" et science du "behavior" ". Vol. 4, n 2, p. 313-326. . «Structure et causalité ». Vol. 3, n 1, p. 3-32.

KATTAN, Naïm. "Ontologie, esthétique et œuvre d'art littéraire». Vol. 5, n 2, p. 261-269.

KERSZBERG, Pierre. «La relativité selon Poincaré. De l'idée au principe ». Vol. 31, n 1, p. 39-56.

KIM, Jaegwon. «L'émergence, les modèles de réduction et le mental». Vol. 27, n 1, p. 11-26.

KIRMAN, Alan. «La pensée évolutionniste dans la théorie économique néoclassique ». Vol. 25, $\mathrm{n}^{\circ}$ 2, p. 219-237.

KISTLER, Max. «Réduction fonctionnelle et réduction logique». Vol. 27, n 1, p. 27-38.

$\mathrm{KOCH}$, Isabelle. «Image plotinienne, image augustinienne». Vol. 25, n 1, p. 73-90.

KOKAY, Victor. «L'axiologie d'Ingarden». Vol. 22, n 1, p. 35-52.

KYMLICKA, Will. «Le libéralisme et la politisation de la culture». Vol. 19, n 2, p. 93-115.

LABARRIERE, Jean-Louis. "Des vertus du décentrement dans Les Ruines de Volney ». Vol. 20, $\mathrm{n}^{\circ} 1$, p. 113-129.

LABSON, Sam. «Dialogue sur l'infinité et la réalité». Vol. 10, n² 2, p. 377-402.

LACHANCE, Michaël. «Déploiement et résistances chez Foucault». Vol. 14, n 1, p. 33-56.

LACHARITÉ, Normand. "Un modèle général pour décomposer la relation d'impact dans les recherches sur l'impact social de la science et de la technologie». Vol. 16, n ${ }^{\circ}$ 1, p. 109-147.

LACROIX, Benoît, et Yvan LAMONDE. "Les débuts de la philosophie universitaire à Montréal. Les Mémoires du doyen Ceslas Forest, o. p. (1885-1970)». Vol. 3, n 1, p. 55-79.

LAFERRIÈRE, Michel. "Les pièges de l'antipsychiatrie ». Vol. 4, n 2, p. 267-276.

LAFLAMME, Simon. "Problématique de philosophies morales occidentales ». Vol. 13, $\mathrm{n}^{\circ} 1$, p. 21-38. . "Sartre et la sociologie. La notion de totalisation». Vol. 10, n 1, p. 53-73.

LAFOREST, Guy. «Le Québec et l'éthique libérale de la sécession». Vol. 19, n² 2, p. 199-214.

LAFRANCE, Jean-David. «L'attitude de tenir une phrase pour vraie et le holisme psycholinguistique». Vol. 31, n² 2, p. 373-392.

LAFRANCE, Yvon. «Aristote et l'analyse géométrique ». Vol. 5, n² 2, p. 271-307.

LAGRÉE, Jacqueline. "Sens et vérité chez Clauberg et Spinoza ". Vol. 29, n 1, p. 121-138.

LAGUEUX, Maurice. "Grandeur et misère du socialisme scientifique ». Vol. 10, n 2, p. 315-340. . «L'arrière-fond philosophique du concept de plan ». Vol. 1, n 1, p. 51-82. . "Rationalité et sélection naturelle en économie ». Vol. 25, n² 2, p. 163-180.

LAMONDE, Yvan, et Benoît Lacroix. "Les débuts de la philosophie universitaire à Montréal. Les Mémoires du doyen Ceslas Forest, o. p. (1885-1970)». Vol. 3, n 1, p. 55-79.

LAMOUREUX, Diane. «Vivons-nous dans un monde post-féministe?» Vol. 21, n 2, p. 321-332.

LANTEIGNE, Josette. «Quelques remarques sur le jugement ». Vol. 19, n 1, p. 25-43. 
LAPOINTE, Sandra. "Introduction : Bernard Bolzano. Contexte et actualité ». Vol. 30, n 1 , p. 3-17.

. Bibliographie. Vol. 30, n 1, p. 235-243.

LAPOINTE, Sandra, et Jacques DUBUCS. «Preuves par excellence». Vol. 30, n 1, p. 219-234.

LAROCHE-PARENT, Madeleine. «La femme (dite barrée) selon l'approche lacanienne ». Vol. 12, nº 1, p. 165-176.

LAURIER, Daniel. «Note sur le puzzle de Kripke». Vol. 15, n 1, p. 31-39. . «Les états intentionnels des créatures solitaires». Vol. 14, n² 2, p. 329-359. . «Remarques sur la sémiotique ». Vol. 11, n 1, p. 91-109.

LAVOIE, Michel. "Le pseudo-féminisme du néo-conservatisme féminin ». Vol. 21, n 2, p. 357-364.

LECLERC, André. «La théorie générale des modes verbaux dans les grammaires philosophiques de l'époque classique ». Vol. 15, n² 2, p. 331-387.

LEGAULT, Georges A. «La parole du philosophe éthicien est-elle crédible ?» Vol. 17, n 1 , p. 21-43.

LEGAULT, Josée. "Les dangers d'une Charte des droits enchâssée pour un Québec indépendant». Vol. 19, n² 2, p. 145-155.

LENOIR, Norbert. «La loi et les deux visages du citoyen chez J.-J. Rousseau ». Vol. 28, $\mathrm{n}^{\circ}$ 2, p. 327-350.

. «Un problème de la légitimité politique dans la pensée de Rousseau ». Vol. 27, n², p. 323-350.

LEPAGE, François. "Qu'est-ce qu’un acte jugé faisable? ». Vol. 28, n² 2, p. 369-380.

. «La question des attitudes propositionnelles et les limites de la sémantique». Vol. 15, n 1 , p. 59-74.

. «La naissance de la théorie des types». Vol. 11, n 2, p. 277-297.

LEROUX, Georges. "Le sujet du souci : à propos de l'Histoire de la sexualité de Michel Foucault». Vol. 14, n 1 , p. 5-32.

LEROUX, Jean. "À propos de "L'explicitation d'un concept". (François Tournier, "L'explication d'un concept”, Philosophiques, vol. 6, n 1, p. 65-118)». Vol. 6, n² 2, p. 273-282.

. "Concept de théorie et contexte diachronique». Vol. 5, n 2, p. 251-259.

LETOCHA, Danièle. «Le trop dit, le dit, le mal dit et le non dit dans le discours althussérien sur l'idéologie». Vol. 9, n 1, p. 41-94.

LÉVESQUE, Claude. «L'inscription de la psychanalyse ». Vol. 4, n² 2, p. 277-286.

LEYDET, Dominique. «Introduction». Vol. 29, n² 2, p. 175-191.

. «Patriotisme constitutionnel et identité nationale». Vol. 19, n 2, p. 81-91.

LIGHT, Steve. «Une ou deux choses à propos de Baudrillard». Vol. 22, nº 1, p. 65-78.

LIVET, Pierre. "Jeux évolutionnaires et paradoxe de l'induction rétrograde (backward induction)». Vol. 25, n² 2, p. 181-201.

. "Structure noématique et transcendance du Dasein». Vol. 20, n² 2, p. 323-346.

LIVINGSTON, Paisley. «Le dilemme de Bratman : problèmes de la rationalité dynamique ». Vol. 20, n 1, p. 47-67. 


\section{Philosophiques / Automne 2004}

LORIES, Danielle. «Art contemporain : questions nouvelles pour l'esthétique ? ». Vol. 23, n 1 , p. 15-35.

LUC, Laurent-Paul. «Vie et vie pensante dans le Systemfragment que Hegel rédigea lors de son séjour à Francfort ». Vol. 24, n² 2, p. 299-311.

. «Le jeune Hegel et la raison en liberté : la positivité de la religion chrétienne ». Vol. 21, $\mathrm{n}^{\circ}$ 1, p. 19-76.

. «Révolution et spéculation chez le jeune Marx». Vol. 10, n² 2, p. 205-220.

. «Le concept de démocratie dans la critique du droit politique hégélien ». Vol. 9, nº 1, p. 119-134.

. «La théorie hégélienne du savoir ». Vol. 7, n 1, p. 55-76.

LY, Igor. "Identité et égalité : le criticisme de Poincaré». Vol. 31, n 1, p. 179-212.

MADISON, Gary Brent. «Le postulat d'objectivité dans la science et la philosophie du sujet ». Vol. 1, n 1, p. 83-139.

MAGGINI, Golfo. "Gestalt, subjectivité, négativité : l'interprétation heideggerienne du Surhomme et sa critique de la morphologie des cultures chez Spengler». Vol. 26, $\mathrm{n}^{\circ} 1$, p. 53-70.

MAINVILLE, Alain, et Ulysses SANTAMARIA. «Marx et le matérialisme : sens et valeur de la première thèse sur Feuerbach». Vol. 14, n 2, p. 381-409. . «Marx : entre l'idéalisme radical et l'individualisme anarchique ». Vol. 11, n² 2, p. 299-333.

MARCIL-LACOSTE, Louise. "Introduction générale : l'impasse des égaux ». Vol. 11, n 1, p. 113-123.

. "Cent quarante manières d'être égaux ». Vol. 11, n 1, p. 125-136.

. «L'héritage cartésien : l'égalité épistémique». Vol. 15, nº 1, p. 77-94.

MARCHAND, Alain Bernard. «Mimèsis et catharsis : de la représentation à la dénégation du réel chez Aristote, Artaud et Brecht». Vol. 15, n 1, p. 107-127.

MARGOT, Jean-Paul. «La lecture foucaldienne de Descartes : ses présupposés et ses implications». Vol. 11, n 1, p. 3-39.

MARION, Mathieu. "Wittgenstein, l'intentionnalité et les règles». Vol. 25, n 1, p. 3-27.

MASSÉ, Sylvie, Chantal THÉRY, Steven MORIN, Bernard OUELLET et Hélène TURCOTTE. «Les autobiographies fœtales masculines ou Jonas dans le ventre de la baleine ». Vol. 21, $\mathrm{n}^{\circ} 2$, p. 503-523.

MASSICOTTE, Guy. "Critique de la pensée historique. Les premiers historiens français du mouvement ouvrier». Vol. 9, n 1, p. 3-39.

MATHIOT, Jean. «Évolution, sélection, information. La question de la convergence». Vol. 25, $\mathrm{n}^{\circ} 2$, p. 203-218.

MATHIOT, Jean, et Robert NADEAU. «Présentation». Vol. 25, n² 2, p. 151-161.

MATTE, Martin. «Jean-Jacques Rousseau et Friedrich Schiller : le théâtre sous le feu des lumières ". Vol. $17, \mathrm{n}^{\circ}$ 2, p. 101-145.

MAWHIN, Jean. «Les fondements de la mécanique en amont et en aval de Poincaré ». Vol. 31, $\mathrm{n}^{\circ} 1$, p. 11-38.

MCCORMICK, Peter. «Le sublime et la fiction». Vol. 23, n 1, p. 93-112. 
. «Sur le développement du concept de l'intentionnalité chez Brentano et Husserl ». Vol. 8, $\mathrm{n}^{\circ} 2$, p. 227-237.

. «L'esthétique de Dilthey : Phénoménologie et théorie littéraire». Vol. 2, nº 2, p. 229-252. . «Heidegger sur le chemin du langage». Vol. 1, n 2, p. 15-36.

MCLAUGHLIN, Brian. "Herméneutique cosmique». Vol. 27, n 1, p. 63-76.

MÉCHOULAN, Éric. "Du bon usage de la haine et du respect dans les Pensées de Pascal». Vol. 24, n² 2, p. 259-275.

MELKEVIK, Bjarne. «Présentation». Vol. 24, n 1, p. 3-7. . «Du contrat à la communication : Habermas critique Rawls». Vol. 24, n 1, p. 59-70.

MENDENHALL, Vance. «Essai sur l'incompétence esthétique». Vol. 10, n² 2, p. 341-359.

MENDONÇA, W. P. «Intelligence artificielle et signification. À propos des limites et des possibilités des sciences cognitives". Vol. 17, n 1, p. 3-19.

MENKE, Christopher. «Le regard esthétique. Affect et violence, plaisir et catharsis ». Vol. 23, $\mathrm{n}^{\circ} 1$, p. 67-79.

MICHEL, Alain. «La réflexion de Poincaré sur l'espace, dans l'histoire de la géométrie ». Vol. 31, $n^{\circ} 1$, p. 89-114.

MIGUELEZ, Roberto. "Kant et la nature». Vol. 23, n² 2, p. 253-264.

. «L'articulation du général et du particulier : une approche méthodologique dans le champ des sciences sociales ». Vol. 11, n 2, p. 251-276.

MOGGACH, Douglas. «Phénoménologie et dialectique du travail». Vol. 15, n² 2, p. 311-329.

MOLINO, Jean. «Pour une histoire de l'interprétation : les étapes de l'herméneutique (I)». Vol. 12, n 1, p. 73-103.

. «Pour une histoire de l'interprétation : les étapes de l’herméneutique (II)». Vol. 12, n² 2, p. 281-314.

MONETTE, Lise. «Le processus contretransférentiel comme travail de deuil». Vol. 4, $\mathrm{n}^{\circ} 2$, p. 305-312.

MONNOYER, Jean-Maurice. "Formes de complexion, types de connexion - Mach, Ehrenfels et Meinong». Vol. 26, n² 2, p. 245-261.

MONTMINY, Jacques. "Origines et fondements philosophiques de la relativité : les conceptions de Mach, Galilée et Einstein». Vol. 22, n 1, p. 21-34.

MONTPETIT, Raymond. «L'esthétique de Rodolphe de Repentigny et la phénoménologie ». Vol. 5, n² 2, p. 211-228.

MORIN, Alain, et James Everett. "Conscience de soi et langage intérieur : quelques spéculations ». Vol. 17, n² 2, p. 169-188.

MORIN, Marie-Josée. "La pensée écoféministe : le féminisme devant le défi global de l'ère techno-scientifique ". Vol. 21, n² 2, p. 365-380.

MORIN, Yvan. "Une double aperception affective et rationnelle de l'homme symbolisant». Vol. 21, n 1, p. 77-105.

MOROSOLI, Michèle. «Les avatars de la différence». Vol. 11, n 2, p. 389-413.

MORSCHER, Edgar. «La définition bolzanienne de l'analyticité logique». Vol. 30, n 1, p. 149-169.

MULLIGAN, Kevin. «Exactitude et bavardage». Vol. 26, n² 2, p. 177-201. 
MULLIGAN, Kevin, et Jean-Pierre Cometti. «Avant-Propos». Vol. 26, n 2, p. 175.

NADEAU, Christian. "Machiavel, domination et liberté politique». Vol. 30, n 2, p. 321-351.

NADEAU, Robert. «L'évolutionnisme : économique de Friedrich Hayek». Vol. 25, n² 2, p. 257-279. . «La philosophie des sciences après Khun ». Vol. 21, n 1, p. 159-189.

. «Problématique de la preuve en épistémologie contemporaine». Vol. 7, nº 2, p. 217-246.

NADEAU, Robert, et Jean Mathiot. «Présentation». Vol. 25, n² 2, p. 151-161.

NADLER, Steven. «Spinoza et le problème juif de la théodicée ». Vol. 29, n 1, p. 41-56.

NDIAYÉ, Aloyse-Raymond. «Le corps-machine et le vrai homme. La controverse entre Arnauld et M. Le Moine, doyen de Vitré». Vol. 24, n² 2, p. 245-258.

NGUYEN, Vinh-De. «Du Discours sur l'inégalité à l'Émile : le parcours anthropologique de Rousseau». Vol. 15, n² 2, p. 405-419.

. «La critique des anthropologies et le Discours sur l'inégalité de J.-J. Rousseau ». Vol. 13, $\mathrm{n}^{\circ} 2$, p. 253-266.

- «Approche épistémologique de la caractérologie selon le point de vue de J.-C. Pariente ». Vol. 12, n 1, p. 3-31.

NICOLAS, Yann. «Les objets culturels». Vol. 26, n 2, p. 301-314.

NIELSEN, Kai. "Après Rawls : la notion de raisonnable comme outil critique ». Vol. 24, n 1 , p. 9-23. . «Le fardeau de la preuve». Vol. 19, n 2, p. 169-189.

NOELTING, Gérald. "Le constructivisme piagétien et la théorie de l'équilibration illustrés par la construction de la notion de proportion ". Vol. 4, n 2, p. 145-194.

NOOTENS, Geneviève. «La nature de la complémentarité entre le raisonnable et le rationnel chez Rawls ». Vol. 24, n 1, p. 25-41.

OGIEN, Ruwen. «Le rasoir de Kant». Vol. 28, n 1, p. 9-25.

PANACCIO, Claude. «Tarski et la suppositio materialis». Vol. 31, n² 2, p. 295-309. . «La notion de croyance : une approche inscriptionnaliste». Vol. 15, n 1, p. 41-58. . «Les qualités selon Stout». Vol. 13, n² 2, p. 237-252.

. «L'intentionnalité comme phénomène linguistique ». Vol. 8, n 2, p. 239-257.

. Présentation d'une série d'articles sur le thème «Qu'est-ce que prouver scientifiquement?» : Actes du colloque, Université du Québec à Trois-Rivières, 10 au 12 octobre $1979 »$. Vol. 7, n 2, p. 213-215.

. «Des phoques et des hommes». Vol. 6, n 1, p. 45-63.

. «Langage ordinaire et langage abstrait chez Guillaume d'Occam». Vol. 1, n² 2, p. 37-60.

PARADIS, André. «De Condillac à Pinel ou les fondements philosophiques du traitement moral». Vol. 20, n 1, p. 69-112.

- «Pouvoir de la culture et culture du pouvoir». Vol. 14, n 1, p. 57-119.

. «Bernard-Henri Levy : Le mal radical ou la philosophie du désespoir ». Vol. 10, n 1, p. 3-14.

. «Individus ou structures : existe-t-il une éthique marxiste?» Vol. 8, n 2, p. 287-302.

PARRET, Herman. "Le plaisir esthétique et la vérité des sens». Vol. 23, n 1, p. 81-92. 
PATAI, Daphné. «Le regard d'ailleurs : les constructions utopiques de la "différence” ». Vol. 21, $\mathrm{n}^{\circ} 2$, p. 525-545.

PELLERIN, René. "Théories et pratiques de la désaliénation ». Vol. 8, n 1, p. 49-74.

PERALDI, François. «La psychanalyse nord-américaine». Vol. 4, n² 2, p. 287-292.

PERRU, Olivier. "Pour une épistémologie du concept d'association chez Émile Durkheim et chez Max Weber». Vol. 27, n² 2, p. 351-376.

- «Le concept d'association et l'unité politique : étude critique chez Saint-Simon, Fourier et Marx». Vol. 26, n 1, p. 83-108.

PESTIEAU, Joseph. «Le pouvoir de l'idéal et l'idéal du pouvoir». Vol. 8, n² 2, p. 259-272. . "Société et politique avec ou sans État». Vol. 6, n² 2, p. 235-252.

PETTIT, Philip. «Deux sources de la moralité ». Vol. 28, n 1, p. 173-203. . «Le non-conséquentialisme et l'universalisabilité ». Vol. 27, n 2, p. 305-322.

PICHÉ, Claude. «Les fictions de la raison pure». Vol. 13, n² 2, p. 291-303.

PIRONET, Fabienne. "Théologie révélée versus théologie philosophique. Siger de Brabant renverse Thomas». Vol. 31, n² 2, p. 311-347.

PLANTE, Robert. "Le phénomène cognitif comme éco-système chez Piaget ». Vol. 4, $\mathrm{n}^{\circ} 2$, p. 245-249.

PLOURDE, Simonne. «Du jeu et du sommeil à la gravité et à l'insomnie». Vol. 13, n² 2, p. 305-332.

POAMÉ, Lazare Marcelin. «Les tâches d'une philosophie pratique à l'âge de la technoscience ». Vol. 25, n 1, p. 91-109.

POIRIER, Pierre. «L'empire contre-attaque : le retour de la réduction psychophysique ». Vol. 27, $\mathrm{n}^{\circ} 1$, p. 39-62.

POISSANT, Louise. «Pour l'immobilisme?». Vol. 12, n 1, p. 133-146.

POLIAKOV, Igor. "L'explicite et l'implicite dans la conception du signe chez Hobbes ». Vol. 17, $\mathrm{n}^{\circ} 2$, p. 23-51.

POUIVET, Roger. "Lukasiewicz : De l'aristotélisme autrichien à l'aristotélisme polonais ». Vol. 26, n² 2, p. 263-277.

POURTOIS, Hervé. «Luttes pour la reconnaissance et politique délibérative ». Vol. 29, n 2, p. 287-309.

PRONOVOST, Gilles. «L'expression et les frontières de la réalité ». Vol. 13, n 1, p. 3-20.

PROUST, Joëlle. «Les conditions de la connaissance de soi ». Vol. 27, n 1, p. 161-186.

PROUX, Benoit. «Pour une érotique philosophique». Vol. 30, n 2, p. 371-389.

PROVENÇAL, Yvon. «Remarques sur la notion de liberté dans l'histoire occidentale et sur son dépassement possible». Vol. 15, n 1, p. 129-139. . "Une analyse de la notion d'objectivité ». Vol. 14, n² 2, p. 361-380.

PROVOST, Marc-A., et Richard L. HOULD. "Éthologie et cybernétique : leur approche de la psychologie». Vol. 7, n² 2, p. 301-319.

RAILTON, Peter. «Kant rencontre Aristote là où la raison rencontre l'appétit ». Vol. 28, n 1, p. 47-67.

RANGER, Philippe. "Théorie de la connaissance, idéologie et camera obscura». Vol. 10, n², p. 255-263. 
RAULET, Gérard. «L'histoire guérie du concept par l'imagination. Place et fonction de l'imagination dans le marxisme contemporain ". Vol. 6, $\mathrm{n}^{\circ}$ 2, p. 211-234.

. "Marxisme et condition post-moderne». Vol. 10, n 2, p. 289-313.

REID, Jeffrey. «Objectivité et discours chez Hegel». Vol. 28, n² 2, p. 351-368.

REINBOLD, Jacques. "Le behaviorisme et les psychologues sociaux». Vol. 4, n 2, p. 335-340.

RENAULT, Marc. «Nouvel essai sur les sandales d'Empédocle». Vol. 3, n 1, p. 33-54.

RICE, Lee C. «La causalité adéquate chez Spinoza ». Vol. 19, n 1, p. 45-59.

ROCHON, Esther. "En hommage aux araignées... et aux femmes». Vol. 21, n 2, p. 441-451.

ROSEN, Stanley. «La théorie excédentaire de l'Être». Vol. 20, n² 2, p. 423-442.

ROUMANES, Jacques-Bernard. «Une alternative à l'exclusion : la dialogie ». Vol. 11, n 2, p. 353-372.

ROY, Jean. "Millénarisme et situationnisme». Vol. 8, $\mathrm{n}^{\circ}$ 1, p. 3-48. . "Modernité et utopie». Vol. 6, n 1, p. 3-44.

ROY, Marie-Andrée. «Les revendications féministes dans le champ religieux québécois : bilan et prospective». Vol. 21, n 2, p. 433-440.

RUSNOCK, Paul. «Qu'est-ce que la représentation ? Bolzano et la philosophie autrichienne». Vol. 30, n 1, p. 67-81.

SAILLANT, Francine. «Femmes et soins dans la société post-moderne». Vol. 21, n 2, p. 381-392.

SAINT-ARNAUD, Jocelyne. «Les définitions aristotéliciennes de la justice : leurs rapports à la notion d'égalité ». Vol. 11, n 1, p. 157-173.

SAJOUS-D'ORIA, Michèle. "Voltaire et l'affaire Calas au théâtre : une vraie cause au service des mythologies révolutionnaires». Vol. 21, n 1, p. 107-123.

SANTAMARIA, Ulysses, et Alain MAINVILLE. «Marx et le matérialisme : sens et valeur de la première thèse sur Feuerbach». Vol. 14, n² 2, p. 381-409.

. "Marx : entre l'idéalisme radical et l'individualisme anarchique ». Vol. 11, n², p. 299-333.

SAUVÉ, Denis. «Wittgenstein et les conditions d'une communauté ». Vol. 28, n² 2, p. 411-432. . "Signification et états mentaux : à propos de "l'antireprésentationnalisme" de Wittgenstein ». Vol. 25, n 1, p. 29-48.

. «La seconde théorie du langage de Wittgenstein». Vol. 22, n 2, p. 213-236.

. «Wittgenstein, Kripke et le paradoxe des règles (I) ». Vol. 20, n 1 , p. 25-46.

. «Wittgenstein, Kripke et le paradoxe des règles (II) ». Vol. 21, n 1, p. 137-157.

. «Le moi-substance : une interprétation de l'“analyse du morceau de cire” de la seconde Méditation ». Vol. 16, $\mathrm{n}^{\circ}$ 1, p. 73-108.

. «Kant, le matérialisme et la psychologie rationnelle». Vol. 14, n² 2, p. 227-261.

. «Règles et langage privé chez Wittgenstein : deux interprétations». Vol. 17, n 1, p. 45-70.

SAVARY, Claude. «Les sciences humaines et l'interprétation». Vol. 7, n² 2, p. 267-299.

SAVOIE, Roger. «La psychiatrie : une machine à faire croire». Vol. 4, n² 2, p. 261-266.

SCHAAR, Marietje Van der. «L'analogie et la vérité selon Brentano». Vol. 26, n 2, p. 203-217.

SCHLEIFER, Michael. «Behaviorisme et psychologie». Vol. 4, n² 2, p. 327-334. 
SCHNIEDER, Benjamin. «Bolzano sur la structure des propositions et le rôle sémantique des propriétés». Vol. 30, n 1, p. 83-103.

SCHULTESS, Daniel. «L'individuation selon Brentano». Vol. 26, n 2, p. 219-230.

SEBESTIK, Jan. «La dispute de Bolzano avec Kant. Fragment d'un dialogue sur la connaissance mathématique». Vol. 30, n 1, p. 47-66.

SEYMOUR, Michel. «Rawls et le droit des peuples». Vol. 26, n 1, p. 109-137.

. "La question nationale : Introduction". Présentation du numéro spécial Une nation peut-elle se donner la Constitution de son choix?, sous la direction de M. Seymour. Vol. $19, n^{\circ} 2$, p. 5-26.

. "Quelques aspects politiques de l'anti-individualisme». Vol. 19, n² 2, p. 63-77.

. «Les énoncés psychologiques à la première personne et le problème de la connaissance de soi ". Vol. 18, n 1, p. 3-23.

. «Les énoncés de croyance et l'énigme de Kripke». Vol. 15, n 1, p. 5-29.

SIEBEL, Malta. «La notion bolzanienne de déductibilité ». Vol. 30, n 1, p. 171-189.

SILVEIRA, Pablo da. "Deux conceptions de la neutralité de l'État». Vol. 23, n² 2, p. 227-251.

SIMONS, Peter. «Bolzano sur les nombres ». Vol. 30, n 1, p. 127-135.

SMITH, Barry . «Kafka et Brentano». Vol. 26, n² 2, p. 349-371.

. «Les objets sociaux». Vol. 26, n² 2, p. 315-347.

SMITH, Michael. «Quelques énigmes concernant le contrôle de soi ». Vol. 27, n² 2, p. 287-304.

SOSOE, Lukas K. «Le contractualisme et la question des nationalités ». Vol. 19, n 2, p. 29-40.

STERLIN, Carlo. "Les contestations contemporaines de la psychiatrie : de l'antipsychiatrie à la néo-psychiatrie ». Vol. 4, n² 2, p. 251-260.

STROUD, Sarah. «À la recherche de la source des normes déontologiques». Vol. 28, $\mathrm{n}^{\circ} 1$, p. 151-171.

. «Déontologisme et droits». Vol. 26, n 1, p. 139-148.

TALIN, Christian. "De l'infanticide en Chine au XVIII" siècle. Les rapports à la "sociologie" de Montesquieu». Vol. 22, n 1, p. 79-93.

TAPPOLET, Christine, et Daniel Weinstock. «Introduction». Vol. 28, n 1, p. 3-8.

TATZEL, Armin. «La théorie bolzanienne de la fondation et de la conséquence ». Vol. 30, n 1 , p. 191-217.

TEXTOR, Mark. «Bolzano sur le temps et la persistance». Vol. 30, n 1, p. 105-125.

THEAU, Jean. «Remarques sur l'épistémologie française et l'épistémologie américaine ». Vol. 3, $n^{\circ} 2$, p. $183-207$. . «Le rapport quantité-qualité chez Hegel et chez Bergson ». Vol. 2, n 1, p. 3-21.

THIERRY, Patrick. «Le Socrate malsain de l'Assemblée nationale : Burke et Rousseau ». Vol. 21, $\mathrm{n}^{\circ} 1$, p. 125-135.

THOMPSON Dennis, et Amy GUTMANN. «Pourquoi la démocratie délibérative est-elle différente?». Vol. 29, n² 2, p. 193-214.

TOCCAFONDI, Fiorenza. «De Karl Bühler à Karl Popper». Vol. 26, n² 2, p. 279-300.

TOURNIER, François. «Un retournement dans la philosophie de la biologie de K. R. Popper». Vol. 18, n 1, p. 61-94. 
. «La fondation de la bioéthique : une perspective épistémologique». Vol. 16, n² 2 , p. 257-291.

. «L'explication d'un concept». Vol. 6, n 1, p. 65-118.

. «Notes sur l'explicitation d'un concept ». Réponse à l'étude critique de Jean Leroux. Vol. 6, n² 2, p. 283-295.

TREMBLAY, Rémi. "L'auto-méditation phénoménologique pour une communauté des philosophes.» Vol. 7, n 1, p. 3-39.

. «Analyse critique de quelques modèles sémiotiques de l'idéologie (I) ». Vol. 17, n 1, p. 71-112.

. «Analyse critique de quelques modèles sémiotiques de l'idéologie (II) ». Vol. 17, n² 2, p. 53-99.

. «Critique de la théorie marxiste de l’État». Vol. 13, n 2, p. 267-289.

VACHET, André. "La dialectique de l'individu et de la collectivité dans la pensée de Marx ». Vol. 2, n 1 , p. 23-53.

VACHON, Gérard. «Un conseil de Freud aux philosophes». Vol. 16, n 1, p. 3-42.

VALCKE, Louis. "Le monisme épistémologique de la science contemporaine». Vol. 1, $\mathrm{n}^{\circ} 2$, p. 3-13.

VALLÉE, Richard. «Compositionnalité et interprétation». Vol. 30, n² 2, p. 353-370.

• «Note sur l'identité intentionnelle». Vol. 26, n 1, p. 37-52.

. «Le problème de l'esprit d'autrui. Discussion de quelques solutions récentes ». Vol. 15, $\mathrm{n}^{\circ} 2$, p. 421-451.

VALOIS, Raynald. «C. G. Jung et le féminin ». Vol. 21, n² 2, p. 393-404.

VASSILIE-LEMENY, Sorin Titus (et Anna-Maria). «Structure et méthode dans la philosophie du sens et de la valeur». Vol. 2, n 1, p. 83-102.

VERNES, Jean-René. «Le principe de Pascal. Hume et la métaphysique ». Vol. 22, n 2, p. 237-246.

VERNES, Paule-Monique. "Les passions de l'âme de Descartes : les limites d'un type d'explication ». Vol. 24, n 2, p. 231-243.

VIDRICAIRE, André. "La vision comme procédé de communication dans le Discours de la Méthode». Vol. 15, n 1, p. 95-105.

VIRVIDAKIS, Stélios. «Les arguments transcendantaux et le problème de la justification de la normativité morale». Vol. 28, n 1, p. 109-128.

VOIZARD, Alain. "Une interprétation de "la signification est l'usage" ". Vol. 28, $\mathrm{n}^{\circ} 2$, p. 395410.

VONARBURG, Elisabeth. «La science-fiction et les hérö̈nes de la modernité ». Vol. 21, n² 2, p. 453-457.

VUILLEMIN, Jules. «Le chapitre IX du De Interpretatione d'Aristote : vers une réhabilitation de l'opinion comme connaissance probable des choses contingentes ». Vol. 10, nº 1, p. 15-52.

WEINSTOCK, Daniel. «Sécurité et démocratie». Vol. 29, n² 2, p. 351-370. . «Libéralisme, nationalisme et pluralisme culturel». Vol. 19, n² 2, p. 117-144.

WEINSTOCK, Daniel, et Christine TAPPOLET. «Introduction». Vol. 28, n 1, p. 3-8. 
WILLIAMS, Melissa S. «Représentation de groupe et démocratie délibérative : une alliance malaisée». Vol. 29, n² 2, p. 215-249.

WUNENBURGER, Jean-Jacques. «Transfiguration et défiguration du corps souffrant. Les métamorphoses de l'idéal de beauté physique dans les arts plastiques ». Vol. 23, n 1, p. 57-66.

YAKIRA, Elhanan. "Spinoza et le problème de l'intentionnalité». Vol. 29, n 1, p. 139-146.

ZAHAVI Dan. «Réduction et constitution chez le dernier Husserl». Vol. 20, n² 2, p. 363-381.

ZANINETTI, Andrea. "L'importance du mécanisme de projection imaginatif au sein de la démarche éthique spinozienne». Vol. 29, n 1, p. 99-105.

ZEPHIR, Jacques J. «Nature et fonction de la mémoire dans À la recherche $d u$ temps perdu». Vol. 17, n² 2, p. 147-168.

\section{Études critiques}

ARCHAMBEAULT, Marc-Fernand. "Michel Morin, L'Amérique du Nord et la culture, Montréal, Hurtubise, $1982 »$. Vol. 12, n 1, p. 211-215.

AUROUX, Sylvain. «François Duchesneau, L'empirisme de Locke, LaHaye, Martinus Nijhoff, $1973 »$. Vol. 1, n 1, p. 147-170.

BARNEY, Rachel. "Louis-André Dorion, Aristote : Les réfutations sophistiques, introduction, traduction et commentaire, Paris, Vrin, $1995 »$. Vol. 25, n 1, p. 111-120.

BEAUSOLEIL, Jocelyn R. "Jean Grondin, Le tournant dans la pensée de Martin Heidegger, Paris, PUF, 1987 ». Vol. 15, n 1, p. 191-209.

BLAIS, François. «La philosophie du droit a-t-elle besoin d'une philosophie des droits? » Étude critique du livre Philosophie du droit de Alain Renaut et Lukas Sosoe (Paris, PUF, 1991). Vol. 21, n 1, p. 241-251.

- «Philosophie du droit et philosophie des droits. Une réplique à la réponse de Renaut et Sosoe ». Vol. 21, n² 2, p. 607-615.

BOUCHAR, Guy. "Du sexisme à la philosophie du sexisme». À propos du livre La raison en procès de Louise Marcil-Lacoste (Montréal, Hurtubise, 1987). Vol. 16, n 1, p. 163-193.

. «L'androgynie comme modèle hétéropolitique ». Étude critique du livre L'un est l'autre. Des relations hommes et femmes de Élisabeth Badinter (Paris, Odile Jacob, 1987). Vol. 15, $\mathrm{n}^{\circ} 1$, p. 210-220.

. «L'a, b, c de la sémiologie». Étude critique du livre Silence, on parle : introduction à la sémiotique de Jurgen Pesot (Montréal, Guérin, 1979). Vol. 7, n² 2, p. 321-375.

BOULAD-AYOUB, Josiane. "Bernard Labrousse, De l'idéologie dominée, Montréal, Nouvelle Optique, $1977 »$. Vol. 7, n 1, p. 85-92.

BRODEUR, Jean-Paul. «L'ouvrage de Robert Hébert ». Étude critique du livre Mobiles du discours philosophique : recherche sur le concept de réflexion de R. Hébert (Montréal, Hurtubise, 1978). Vol. 6, n 1, p. 131-145.

CANTIN, Serge. " Richard Gervais, Dialectique et totalitarisme, Montréal, Hurtubise, 1990 ». Vol. 18, n², p. 171-176.

CHARRON, Ghyslain. "Jean Roy, Hobbes et Freud, Halifax, Dalhousie University Press, 1976 ». Vol. $4, n^{\circ} 1$, p. 35-49. 
- «Linguistique, philosophie du langage et épistémologie. Réponse à l'étude critique de Pierre Martin». Vol. 3, n 2, p. 261-278.

CLOUTIER, Yvan. «Marc Chabot et André Vidricaire (éd.), Objet pour la philosophie, Québec, Éditions Pantoute, $1983 »$. Vol. 12, n² 2, p. 421-428.

CORREIA, Fabrice. "Théodore Sider, Four-Dimensionalism. An Ontology of Persistence and Time, Oxford, Clarendon Press, $2003 »$. Vol. 31, n 1, p. 239-249.

DORION, Louis-André. «La dialectique d'Aristote dénaturée ». Étude critique du livre La dialectique aristotélicienne. Les principes clés des Topiques de Yvan Pelletier (Montréal, Bellarmin, 1991). Vol. 20, n² 2, p. 485-502.

DUFOUR, Mario. "Claude Lévesque, Le proche et le lointain. Essais, Montréal, VLB, 1994 ». Vol. 23, n 2, p. 427-438.

DUMONT, Fernand. «Une contribution à l'histoire de la philosophie au Québec ». Étude critique du livre La philosophie et son enseignement au Québec 1665-1920 de Yvan Lamonde (Montréal, Hurtubise, 1980). Vol. 10, n 1, p. 119-125.

DUMOUCHEL, Paul. "Émotion et perception». Étude critique du livre Émotions et valeurs de Christine Tappolet (Paris, PUF, 2000). Vol. 29, n 1, p. 371-377.

. «François Duchesneau, Philosophie de la biologie, Paris, PUF, 1997 ». Vol. 26, n 1, p. 149-154.

FERLAND, Madeleine. "Marc Henry Soulet, Le silence des intellectuels. Radioscopie de l'intellectuel québécois, Montréal, Éd. Saint-Martin, 1987 ». Vol. 17, n 1, p. 143-149.

FISETTE, Denis, et Pierre POIRIER. «Réponse à Don Ross ». Vol. 30, n 1, p. 256-262.

FOISY, Suzanne. "Homo Communicativus». Étude critique du livre Homo Æstheticus. L'invention du goût à l'âge démocratique de Luc Ferry (Paris, Grasset, 1990). Vol. 17, n² 2, p. 189-200.

GAGNON, Claude. «Réal Rodrigue, Défaire l'histoire, Longueuil, Préambule, 1980 ». Vol. 10, $\mathrm{n}^{\circ} 1$, p. 111-117.

. "Serge Lusignan, Préface au Speculum Majus de Vincent de Beauvais : réfraction et diffraction, Montréal : Bellarmin; Paris : Vrin, 1979». Vol. 8, n 1, p. 131-137.

GAGNON, Maurice. "François Duchesneau, Genèse de la théorie cellulaire, Montréal : Bellarmin; Paris : Vrin, 1987 ». Vol. 16, n² 2, p. 395-404.

- «Roberto Miguelez, La comparaison interculturelle, Montréal, Presses de l’Université de Montréal, 1977 ». Vol. 6, n² 2, p. 297-307.

GERAETS, Théodore F. "Merleau-Ponty selon Madison ». Étude critique du livre La Phénoménologie de Merleau-Ponty. Une recherche des limites de la conscience de Gary Brent Madison (Paris, Klincksieck, 1973). Vol. 2, n 1, p. 113-123.

. «Réponse à Gary Brent Madison». Vol. 2, n 1, p. 128-130.

GOMBAY, André. "Georges-A. Legault, La structure performative du langage juridique». Vol. 5, n², p. 309-316.

GOULET, Jean. "Guy Lafrance, La philosophie sociale de Bergson. Sources et interprétation, Ottawa, Éd. de l'Université d'Ottawa, 1974 ». Vol. 3, nº 1, p. 81-92.

GRILLO, Éric. "Martin Montminy, Les fondements empiriques de la signification, Montréal : Bellarmin; Paris : Vrin, $1998 »$. Vol. 27, n 1, p. 187-201. 
HÉBERT, Robert. "Christian Atias, Savoir des juges et savoir des juristes. Mes premiers regards sur la culture juridique québécoise, Montréal, Centre de recherche en droit privé et comparé du Québec, 1990 ». Vol. 19, n 1, p. 123-129.

- «Sans trop mâcher les mots, percevoir ». Étude critique du livre Réjean Ducharme, Nietzsche et Dionysos de Renée Leduc-Park (Québec, Presses de l'Université Laval, 1982). Vol. 11, n 1, p. 191-202.

. «D'une falaise d'où l'on voit poindre le soleil de la culture savante ». Contribution au premier cahier de l'Institut québécois de recherche sur la culture (1). Vol. 9, n 2, p. 281-293.

. "D'une falaise d'où l'on voit poindre le soleil de la culture savante ». Contribution au premier cahier de l'Institut québécois de recherche sur la culture (2). Vol. 10, nº 1, p. 97-110.

. «Théodore F. Gerates (dir.), Rationalité N d'un colloque sur les N rationalités ». Étude critique de Rationality To-day/La rationalité aujourd'hui (Ottawa, Presses de l'Université d'Ottawa, 1979). Vol. 8, n 1, p. 139-148.

«Roland Houde, Histoire et philosophie au Québec. Anarchéologie du savoir historique.» Vol. 7, n 1, p. 93-100.

KARGER, Elisabeth. "Sémantique et nominalisme». Étude critique du livre Les mots, les concepts et les choses (la sémantique de Guillaume d'Occam et le nominalisme d'aujourd'hui) de Claude Panaccio (Montréal : Bellarmin; Paris : Vrin, 1992). Vol. 21, n², p. 563-576.

LACHANCE, Michaël. «Georges Leroux et Michel Van Schendel (dir.), Sédiments 1986 : un recueil annuel d'écriture et de réflexion, Montréal, Hurtubise, 1986 ». Vol. 14, n 1, p. 193-202.

. «Il y a toujours un mort celé dans le miroir : structure spéculaire et métaphores tragiques dans D’un miroir et de quelques éclats de Pierre Gravel ». Étude critique du livre D’un miroir et de quelques éclats de Pierre Gravel (Montréal, L’Hexagone, 1985). Vol. 13, $\mathrm{n}^{\circ} 2$, p. 369-382.

. «Nicolas Machiavel, Le Prince, présentation et traduction par Gérald Allard (Le Griffon d'Argile, 1984) »; " Le Prince et autres écrits politiques, présentation de Philippe Ranger, trad. de J. V. Périès revue par P. Ranger (Balises, 1982) ». Vol. 12, n 2, p. 429-443.

LACHARITÉ, Normand. "Vers une ontologie "naturalisée" de l'information». Étude critique du livre The Nature of Information de Paul Young (New York, Praeger, 1987). Vol. 20, $n^{\circ} 2$, p. 473-483.

. "Michel Freitag, Dialectique et société [t. I] : Introduction à une théorie générale du symbolique, et [t. II] : Culture, pouvoir, contrôle. Les modes de reproduction formels de la société, Montréal, Saint-Martin, 1986 ». Vol. 14, n 1, p. 183-192.

LAFRANCE, Guy. «Lire Bergson». Réponse à Jean Goulet. Vol. 3, n 2, p. 279-284.

LAFRANCE, Yvon. "Volonté et liberté». Étude critique du livre Plotin: Traité sur la liberté et la volonté de l'Un [Énnéade VI, 8 (39)] de Georges Leroux, intro., textes grecs, trad. et commentaire de G. Leroux, (Paris, Vrin, 1990). Vol. 20, n 1, p. 189-197.

LAGUEUX, Maurice. «Réponse à Christian Nadeau ». Vol. 30, n 2, p. 445-447.

. "Claude Savary et Claude Panaccio (dir.), L'idéologie et les stratégies de la raison, Ville LaSalle, Hurtubise, 1984 ». Vol. 13, n 1, p. 170-177.

. «Un "bilan” vite parcouru. Réponse à Gérard Raulet. Un bilan du marxisme? » Vol. 13, $\mathrm{n}^{\circ} 1, \mathrm{p} .153-155$. 
LANGLOIS, Luc. «L'universalité du verbum interius ». Études critique du livre L'universalité de l’herméneutique de Jean Grondin (Paris, PUF, 1993). Vol. 22, n 1, p. 137-157.

LEGAULT, Georges A. «Réponses au commentaire de M. André Gombay sur La structure performative du langage juridique». Vol. 5, $\mathrm{n}^{\circ}$ 2, p. 317-329.

LEROUX, Georges. «Alain Boutot, Heidegger et Platon. Le problème du nibilisme, Paris, PUF, $1987 »$. Vol. 19, n 1, p. 111-122.

. «Léonce Paquet, Platon. La Médiation du Regard, Leyden, Brill, 1973 ». Vol. 2, n² 2, p. 253-285.

LETOCHA, Danièle. «Du lieu de l'homme à l'absence de l'homme ». Étude critique du livre L'anthropologie en l'absence de l'homme de Fernand Dumont (Paris, PUF, 1981). Vol. 9, $\mathrm{n}^{\circ} 2$, p. 295-305.

MADISON, Gary Brent. «Réponse à Th. F. Geraets». Vol. 2, n 1, p. 123-128.

. «Sur l'interprétation de Merleau-Ponty de Th. F. Geraets». Étude critique du livre Vers une nouvelle philosophie transcendantale. La genèse de la philosophie de Maurice Merleau-Ponty jusqu'à la phénoménologie de la perception de Théodore F. Geraets (LaHaye, Martinus Nijhoff, 1971). Vol. 2, n 1, p. 103-112.

MARCIL-LACOSTE, Louise. «L'art du malentendu : une réponse à Guy Bouchard». Vol. 16, $\mathrm{n}^{\circ} 1$, p. 195-198.

MARION, Mathieu. «De la logique interne». Étude critique du livre De la logique interne de Yvon Gauthier (Paris, Vrin, 1991). Vol. 21, n 1, p. 213-239.

. «Yvon Gauthier, De la logique interne, Paris, Vrin, 1991 ». Vol. 22, n² 2, p. 301-336.

MARTIN, Pierre. "Ghyslain Charron, Du langage. Confrontation d'une approche structurale et d'une approche phénoménologique: A. Martinet et M. Merleau-Ponty (Ottawa, Éd. de l'Université d'Ottawa, 1972)». Vol. 3, n² 2, p. 255-260.

MENDENHALL, Vance. "Normand Lacharité, Introduction à la méthodologie de la pensée écrite, Montréal, Presses de l'Université du Québec, 1987 ». Vol. 15, n² 2, p. 465-482.

MEUNIER, Jean-Guy. «La logique illocutoire : ses fondements selon Searle et Vanderveken ». Étude critique du livre Foundations of Illocutionay Logic de John Searle et Daniel Vanderveken (Cambridge University Press, 1985). Vol. 13, n² 2, p. 383-402.

. «Sur le matérialisme du jeune Marx». Réponse à l'étude critique de François Tournier. Vol. 13, n 1, p. 164-169.

MICHON, Cyrille. "Occam Redivivus». Étude critique du livre Les mots, les concepts et les choses (la sémantique de Guillaume d'Occam et le nominalisme d'aujourd'hui) de Claude Panaccio (Montréal : Bellarmin; Paris : Vrin, 1992). Vol. 21, n² 2, p. 577-597.

MIGUELEZ, Roberto. «Narration, connaissance et identité chez Paul Ricœur ». Étude critique du livre Temps et récit de Paul Ricœur, Tomes 1, 2 et 3 (Paris, Seuil, 1983-84-85). Vol. 14, $\mathrm{n}^{\circ} 2$, p. $425-433$.

MONETTE, Lise. «D'une lecture coupable de tout ce qu'elle laisse choir... ». Étude critique du livre Freud et le problème de la culpabilité de Ghislain Charron (Ottawa, Éditions de l’Université d'Ottawa, 1979). Vol. 7, n² 2, p. 377-382.

MONTMINY, Martin. «Réponse à Éric Grillo». Vol. 27, n 1, p. 203-206.

MONTPETIT, Raymond. «Réponses au commentaire de Claude Panaccio : Comment parler de la littérature?». Vol. $5, \mathrm{n}^{\circ} 1, \mathrm{p} .173-177$. 
MOSQUERA, Gerardo. "Yvon Lafrance, Pour interpréter Platon II. La ligne en République VI, 509d-511e. Le texte et son histoire, Montréal, Bellarmin, $1994 »$. Vol. 24, n 1, p. 161-183.

NADEAU, Christian. "Maurice Lagueux, Actualité de la philosophie de l'histoire. L'histoire aux mains des philosophes, Québec, Les Presses de l'Université Laval, 2001 ». Vol. 30, $\mathrm{n}^{\circ} 2$, p. 425-444.

NARBONNE, Jean-Marc. "Stanley Rosen, The Question of Being. A Reversal of Heidegger, New Haven/London, Yale University Press, 1993 ». Vol. 23, n 1, p. 163-171.

ONG-VAN-CUNG, Kim Sang. "Jean-Pierre Cléro, Théorie de la perception. De l'espace à l'émotion, Paris, PUF, $2000 »$. Vol. 28, n 2, p. 433-442.

PANACCIO, Claude. "Guillaume d'Occam et la sémantique des concepts. Réponse à Elisabeth Karger et Cyrille Michon». Vol. 21, n 2, p. 599-605.

. «Raymond Montpetit, Comment parler de la littérature?». Vol. 5, n 1, p. 155-171

PHILIE, Patrice. "Crispin Wright, Saving the Differences: Essays on Themes from Truth and Objectivity, Cambridge, Harvard University Press, 2003 ». Vol. 31, nº 2, p. 417-429.

PIOTTE Jean-Marc. «Note. Réponse à l'étude critique de L.-M. Vacher ». Vol. 6, n 2, p. 324-326.

POIRIER, Pierre, et Denis FISETTE. «Réponse à Don Ross». Vol. 30, n 1, p. 256-262.

PORSET, Charles. "L'efficacité du symbolique : Lumières et Révolution». Étude critique du livre Contre nous de la tyrannie... Des relations idéologiques entre Lumières et Révolution de Josiane Boulad-Ayoub (Montréal, Hurtubise, 1989). Vol. 20, nº 1, p. 177-188.

RATTÉ, Michel. «Rainer Rochlitz, L'art au banc d'essai. Esthétique et critique, Paris, Gallimard, 1998 ». Vol. 27, n 2, p. 425-442.

RAULET, Gérard. «Un bilan du marxisme? ». Étude critique du livre Le marxisme des années soixante : Une saison dans l'histoire de la pensée critique de Maurice Lagueux (Ville LaSalle, Hurtubise, 1982). Vol. 13, n 1, p. 147-152.

REBOUL, Olivier. Étude critique du livre La théologie kantienne pré-critique de P. Laberge (Ottawa, Éd. de l'Université d'Ottawa, 1973). Vol. 1, n 2, p. 83-93.

RENAUD, François. «Jean Grondin, Hans-Georg Gadamer. Eine Biographie, Tübingen, Mohr Siebeck, $1999 »$. Vol. 28, n 1, p. 205-218.

RENAUT, Alain, et Lukas K. SOSOE. «La philosophie du droit. Réponse à François Blais». Vol. 21, n 1, p. 253-257.

ROBERT, Serge. "Les ouvrages de Yvon Gauthier». Étude critique du livre Fondements des mathématiques: Introduction à une philosophie constructiviste de Y. Gauthier (Montréal, Les Presses de l'Université de Montréal, 1976); et de Méthodes et concepts de la logique formelle de Y. Gauthier (Montréal, Presses de l’Université de Montréal, 1978). Vol. 6, n 1, p. 119-130.

ROCHLITZ, Rainer. «Réponse à Michel Ratté ». Vol. 27, n² 2, p. 443-448.

ROSS, Don. «L'étude de tout, par tous les moyens ». Étude critique du livre Philosophie de l'esprit, état des lieux de Denis Fisette et Pierre Poirier (Paris, Vrin, 2000). Vol. 30, n 1 , p. 245-255.

ROY, Bruno. «Claude Gagnon : De la cabale des philosophes à la littérature des cabalistes ». Étude critique de plusieurs ouvrages de Claude Gagnon. Vol. 7, n 1, p. 77-84.

SAUVÉ, Denis. Étude critique du livre La cohérence de la doctrine kantienne de la liberté de B. Carnois (Paris, Seuil, 1973). Vol. 1, n² 2, p. 95-110. 
SOSOE, Lukas K., et Alain RENAUT. «La philosophie du droit. Réponse à François Blais ». Vol. 21, nº 1, p. 253-257.

TAPPOLET, Christine. "Les émotions et leurs conditions d'adéquation. Réponse à Paul Dumouchel ». Vol. 29, n² 2, p. 378-382.

THÉRIEN, Gilles. "Claude Lagadec, Dominances. Essai de sociobiologie sur l'inégalité et la tromperie, Longueuil, Le Préambule, 1983 ». Vol. 11, nº 2, p. 415-421.

THIBAULT, Pierre. "L'histoire du thomisme québécois ou de l'importance de mettre les âmes à leur place». Vol. $8, \mathrm{n}^{\circ}$ 2, p. 343-348.

TOURNIER, François. "Jean-Guy Meunier, Genèse du matérialisme dans les écrits de jeunesse de Karl Marx, Ottawa, Éd. de l’Université d'Ottawa, 1981 ». Vol. 13, n 1, p. 155-164.

TROUILLARD Jean. "Luc Brisson, Le Même et l'Autre dans la structure ontologique du "Timée", de Platon. Un commentaire systématique du "Timée" de Platon, Paris, Klincksieck, $1974 »$. Vol. 2, n² 2, p. 287-299.

TURGEON, Marc. "Claude Lévesque, Dissonance. Nietzsche à la limite du langage, Montréal, Hurtubise, $1988 »$. Vol. 18, n 1, p. 169-174.

VACHER, Laurent-Michel. "Jean-Marc Piotte, Marxisme et pays socialistes, Montréal, VLB éditeur, 1979». Vol. 6, n² 2, p. 309-323.

VIDRICAIRE, André. "Robert Hébert, L’Amérique française devant l'opinion étrangère 17561960. Anthologie, Montréal, l'Hexagone, $1989 »$. Vol. 18, nº 2, p. 159-169.

\section{Disputationes}

DEVIDI, David. «Sur la preuve de consistance de Gauthier et le programme de Frege ». Vol. 31, $\mathrm{n}^{\circ} 1$, p. 215-220.

GAUTHIER, Yvon. «Précis de Internal Logic. Foundations of Mathematics from Kronecker to Hilbert, (Dordrecht, Kluwer, 2002)». Vol. 31, n 1, p. 213-214.

. «Réponses à mes critiques ». Réponse à David Devidi, Mathieu Marion, et Zach Richard. Vol. 31, n 1, p. 231-238.

LARMORE, Charles. "Le normatif et l'évaluatif». Vol. 31, nº 2, p. 398-402.

LAURIER, Daniel. «Entre la rime et la raison. Précis de L'esprit et la nature, Montréal, Presses de l’Université de Montréal, 2002 ». Vol. 30, n² 2, p. 407-410

- «Réponses à mes critiques ». Réponse à Martin Montmigny et Alfredo Paternoster. Vol. 30, n² 2, p. 421-424.

LIVET, Pierre. « Principes normatifs ou pragmatiques, et pondération des arguments ». Vol. 31, $\mathrm{n}^{\circ} 2$, p. 402-405.

MARION, Mathieu. "Fondements ou constructivité ?" Étude critique du livre Internal Logic. Foundations of Mathematics from Kronecker to Hilbert de Yvon Gauthier (Dordrecht, Kluwer, 2002). Vol. 31, n 1, p. 225-230.

MONTMINY, Martin. «À propos d'une objection contre le naturalisme modéré ». Étude critique du livre L'esprit et la nature de Daniel Laurier (Montréal, Presses de l'Université de Montréal, 2002). Vol. 30, n² 2, p. 411-415. 
OGIEN, Ruwen. "Précis de Le rasoir de Kant et autres essais de philosophie pratique, Paris, Éditions de l'Éclat, $2003 »$. Vol. 31, n² 2, p. 393-398. . «Réponses à mes critiques». Vol. 31, n² 2, p. 408-416

PATERNOSTER, Alfredo. "La vexante question du normatif». Étude critique du livre L'esprit et la nature de Daniel Laurier (Montréal, Presses de l'Université de Montréal, 2002). Vol. $30, n^{\circ} 2$, p. 417-419.

STROUD, Sarah. «Rationalité, humanité, normativité ». Vol. 31, n² 2, p. 405-408.

$\mathrm{ZACH}$, Richard. «Le quantificateur effini, la descente infinie et les preuves de consistance de Gauthier». Étude critique du livre Internal Logic. Foundations of Mathematics from Kronecker to Hilbert de Yvon Gauthier (Dordrecht, Kluwer, 2002). Vol. 31, nº 1, p. 221-224.

\section{Comptes rendus}

BAERTSCHI, Bernard. "André Duhamel, Une éthique sans point de vue moral. La pensée éthique de Bernard Williams, Québec, Presses de l’Université Laval, 2003 ». Vol. 31, n 2, p. 433-435.

BAUGH, Bruce. «Philip Knee, Qui perd gagne : essai sur Sartre, Québec, Presses de l’Université Laval, $1993 »$. Vol. 22, n 1, p. 159-163.

BEAULIEU, Alain. "Véronique Bergen, L'ontologie de Gilles Deleuze, Paris, L'Harmattan, $2001 »$. Vol. $29, n^{\circ} 1$, p. 165-167.

. "Alain Badiou, Court traité d'ontologie transitoire (Paris, Seuil, 1998) ». "Alain Badiou, Petit manuel d'inesthétique (Paris, Seuil, 1998)». "Alain Badiou, Abrégé de métapolitique (Paris, Seuil, 1998)». Vol. 27, n 1, p. 207-211.

. "Alain Badiou, Saint Paul. La fondation de l'universalisme, Paris, PUF, 1997 ». Vol. 26, $\mathrm{n}^{\circ} 2$, p. 373-375.

. "Laurent Van Eynde, Introduction au romantisme d'Iéna. Friedrich Schlegel et l'Athenäum, Bruxelles, Ousia, $1997 »$. Vol. 26, n 1, p. 157-159.

BEAUSOLEIL, Jocelyn R. "Georg Wilhelm Friedrich Hegel, Fragments de la période de Berne (1793-1796), Paris, Vrin, $1987 »$. Vol. 16, n 1, p. 201-205.

BELISLE, Marc. "Michel Morin, Mort et résurrection de la loi morale, Montréal, Hurtubise, 1997 ». Vol. 28, n 1, p. 223-226.

BELLAVANCE, Guy. «Niklas Luhmann, Amour comme passion. De la codification de l'intimité, trad. de l'all. par Anne-Marie Lionnet (Aubier, 1990)». "Niklas Luhmann, Ecological Communication, trans. by John Bednarz Jr. (The University of Chicago Press, 1989)». Vol. 18, n², p. 177-180.

BELZILE, Jean-François. "Michel Hulin, Öaôkara et la non-dualité, Bayard, Paris, 2001 ». Vol. 30, n² 2, p. 455-457.

- "Marc Ballanfat, Les matérialistes dans l'Inde ancienne, trad., notes et commentaires; préface de Pierre-Sylvain Filliozat, Paris, L'Harmattan, 1997 ». Vol. 25, n 1, p. 127-129.

BERGERON, Fernand-Luc. "Anne Cauquelin, La mort des philosophes et autres contes, Paris, PUF, $1992 »$. Vol. 23, n 1, p. 173-174.

BERNIER, Paul. «Hilary Putnam, Représentation et réalité, trad. de Claudine Engel-Tiercelin, Paris, Gallimard, 1990 ». Vol. 18, n² 2, p. 191-195. 
BILODEAU, Renée. "Donald Davidson, Paradoxes de l'irrationalité, trad. de Pascal Engel, Combas, Éditions de l’Éclat, $1991 »$. Vol. 20, n² 2, p. 503-506.

BLACKBURN, Pierre. "Jon Elster, Le laboureur et ses enfants : deux essais sur les limites de la rationalité, Paris, Minuit, $1986 »$. Vol. 15, n² 2, p. 489-493.

BLANCHARD, François. "Cahiers de recherche sociologique, vol. I, septembre 1983, "Connaissance et société”, Montréal, Université du Québec à Montréal, Collectif du Département de sociologie». Vol. 11, n 2, p. 423-425.

BLANCHARD, Martin. «Bernard Baertschi et Kevin Mulligan (dir.), Les nationalismes, Paris, PUF, $2002 »$. Vol. 31, n 1, p. 254-258.

Bodéüs, Richard. «Bertrand Dumoulin, Analyse génétique de la Métaphysique d'Aristote, Montréal : Bellarmin; Paris : Les Belles-Lettres, 1986 ». Vol. 17, n 1, p. 154-156.

. «E. Robillard, Justin. L'itinéraire philosophique, Montréal : Bellarmin; Paris : Cerf, $1989 »$. Vol. $17, \mathrm{n}^{\circ} 1$, p. 156-157.

. "Jean Frère, Les Grecs et le désir de l'être. Des Préplatoniciens à Aristote, Paris, BellesLettres, $1981 »$. Vol. 16, n 1, p. 209-210.

BONIN, Pierre-Yves. «Ronald Dworkin, "Assisted Suicide : What the Court Really Said", The New York Review of Books, 25 septembre 1997 ». Vol. 25, n² 2, p. 306-311.

BORDELEAU, Léo-Paul. "Marc Renault, Le singulier. Essai de monadologie, Montréal : Bellarmin; Paris : Desclée, $1979 »$. Vol. 9, n² 2, p. 355-359.

BOSS, Gilbert. «Michel Cornu, Patience du détachement, Lausanne, L'Âge d'homme, 1989 ». Vol. 17, n 1, p. 164.

BOUCHARD, Guy. "Danielle Lories, Expérience esthétique et ontologie de l'œuvre, Bruxelles, Palais des académies, 1989». Vol. 19, n 1, p. 155-157.

. "Pierre Gravel, Pour une logique du sujet tragique. Sophocle, Montréal, Presses de l’Université de Montréal, $1980 »$. Vol. 8, n² 2, p. 358-360.

BOULAD-AYOUB, Josiane. "Albert Soboul, Dictionnaire historique de la Révolution française, Paris, PUF, $1989 »$. Vol. 17, n² 2, p. 212-214.

. «Martial Guéroult, Histoire de l'histoire de la philosophie, Livre I, tome II, En Allemagne, de Leibniz à nos jours, 1988, p. 329-673; livre I, tome III, En France, de Condorcet à nos jours, p. 674-1084. Paris, Aubier». Vol. 16, n² 2, p. 439-443.

. «René Zapata, La philosophie russe et soviétique, Paris, PUF, 1988 ». Vol. 16, n² 2, p. 443-445.

. «Venant Cauchy (dir.), Philosophie et culture, Actes du XVII congrès mondial de philosophie/Philosophy and Culture, Proceedings of the XVII World Congress of Philosophy, 5 tomes, Montréal, Beffroi, 1986-1987». Vol. 16, n² 2, p. 436-439.

. "Collectif. Nation, Souveraineté, Droit: la question nationale. Actes du IVe Colloque interdisciplinaire de la Société de philosophie du Québec, Montréal : Bellarmin; Paris : Desclée, $1980 »$. Vol. 9, n² 2, p. 342-344.

. "Julien J. Lafontant, Montesquieu et le problème de l'esclavage dans L'Esprit des lois, Sherbrooke, Éd. Naaman, 1979 ». Vol. 8, n² 2, p. 349351.

. "Jim Macadam, Michaël Neuman et Guy Lafrance, Trent Rousseau Papers; Études Rousseau - Trent, Ottawa, Éditions de l'Université d'Ottawa, 1980 ». Vol. 8, nº 1, p. 204. 
. «Julien Bigras, Le choc des ceuvres d'art, Montréal, Hurtubise, 1980 ». Vol. 8, n 1, p. 211-214.

CARON, Anita. "Jean-Claude Breton, Foi en soi et confiance fondamentale. Dialogue entre Marcel Légaut et Erik H. Erikson, Montréal : Bellarmin; Paris : Cerf, 1987». Vol. 15, n² 2, p. 493-495.

CARON, Richard. "Yvan Lamonde, Gens de parole. Conférences publiques, essais et débats à l'Institut canadien de Montréal 1845-1871, Montréal, Boréal, 1990 ». Vol. 20, n 1, p. 199-201.

CARRIER, André. "Gilles Lane, Si les marionnettes pouvaient choisir. Recherche sur les droits, l'obligation morale et les valeurs, Montréal, L’Hexagone, 1983 ». Vol. 12, n² 2, p. 451-454.

CASTELNÉRAC, Benoît. "Catherine Collobert (dir.), L'avenir de la philosophie est-il grec? Textes présentés au cours d'un colloque à Ottawa, dans le cadre du $67^{\mathrm{e}}$ Congrès de l'Association canadienne-française de l'avancement des sciences (mai 1999), Montréal, Fidès, $2002 »$. Vol. $30, n^{\circ} 1$, p. 272-275.

. «Monique Canto-Sperber, Éthiques grecques, Paris, PUF, 2001 ». Vol. 30, n 1, p. 268-271.

. «Platon, La République, intro., trad., et notes de Georges Leroux, Paris, Flammarion, $2002 »$. Vol. 29, n 2, p. 407-411.

CHARBONNEAU, Marie-Andrée. «Henri F. Ellenberger, Histoire de la découverte de l'inconscient, trad. J. Feisthauser, présentation d‘Élizabeth Roudinesco et complément bibliographique par Olivier Husson, Paris Fayard, 1994 ». "Henri F. Ellenberger, Médecines de l'âme. Essais d'histoire de la folie et des guérisons psychiques, textes réunis et présentés par Élizabeth Roudinesco, Paris, Fayard, 1995 ». Vol. 27, n² 2, p. 449-456.

CHARLAND, Mario. "Michel Haar, La philosophie française entre phénoménologie et métaphysique, Paris, PUF, 1999 ». Vol. 29, n² 2, p. 402-407.

. «Françoise Dastur, Husserl. Des mathématiques à l’histoire, Paris, PUF, 1999 ». Vol. 28, $\mathrm{n}^{\circ} 2$, p. $443-446$.

CHARLES, Sébastien. «Bernard Mandeville, Pensées libres sur la religion, sur l'Église, et sur le bonheur national, seconde édition revue, corrigée et augmentée. Manuscrit Montbret 475 de la Bibliothèque municipale de Rouen, trad. de l'anglais et édité par P. Carrive et L. Carrive, avec intro. de P. Carrive, Paris, Honoré Champion, 2000 ». Vol. 30, n 2, p. 482-484.

. "Geneviève Brykman, (dir.), Ressemblance et dissemblances dans l'empirisme britannique, Nanterre, Publications du Département de philosophie, Paris X-Nanterre, 1999». Vol. 26, $\mathrm{n}^{\circ} 2$, p. 376-379.

«Marcel Conche, Le sens de la philosophie, La Versanne, Encre Marine, 1999 ». "Marcel Conche, Le destin de solitude, La Versanne, Encre Marine, 1999 ». Vol. 26, n² p. 380-383.

CHARLES, Syliane. "Kim Sang Ong-Van-Cung, Descartes et l'ambivalence de la création, Paris, Vrin, $2000 »$. Vol. 29, n 2, p. 400-402.

CHAUVIER, Stéphane. "Daniel Tanguay, Léo Strauss. Une biographie intellectuelle, Paris, Grasset, $2003 »$. Vol. 31, n 2, p. 431-433.

CHRÉTIEN, Émile. "Pierre du Calvet, Appel à la justice de l’État, extraits présentés par Jean-Paul de Lagrave et Jacques-G. Ruelland, Le Griffon d'Argile, $1986 »$. Vol. 14, n² 2, p. 437-440. 


\section{Philosophiques / Automne 2004}

CHUNG, Ryoa. «Jean-Claude Guillebaud, Le Principe d'humanité, Paris, Seuil, 2001 ». Vol. 29, $\mathrm{n}^{\circ}$ 2, p. 396-400.

CLOUTIER, Yvan. " "La naissance de la psychanalyse à Montréal”, in Frayages, n 3 (1987), Montréal, Éd. Frayages, $1987 »$. Vol. 15, n 1, p. 221-225.

. "Jacques Beaudry, Autour de Jacques Lavigne, philosophe. Histoire de la vie intellectuelle d'un philosophe québécois de 1935 à aujourd'hui accompagnée d'un choix de textes de Jacques Lavigne, Trois-Rivières, Éd. du Bien public, 1985 ». Vol. 13, n² 2, p. 429-431.

CLOZE, Marie-Claire. "Bertrand Russell, Écrits de logique philosophique, avant-propos et trad. de l'anglais par Jean-Michel Roy, Paris, PUF, $1989 »$. Vol. 17, n² 2, p. 217-221.

CONSTANTINEAU, Philippe. "Catherine Collobert, L'Être de Parménide ou le refus du temps, préf. de Marcel Conche, Paris, Kimé, 1993 ». Vol. 22, n 1, p. 163-165.

CORBEIL, Yvon. «Jeffrey Andrew Barash, Heidegger et son siècle, Paris, PUF, 1995 ». Vol. 25, $\mathrm{n}^{\circ} 2$, p. 288-293.

. "Jean Greisch, Ontologie et temporalité. Esquisse d'une interprétation intégrale de Sein und Zeit, Paris, PUF, 1994 ». Vol. 23, n² 2, p. 451-454.

CÔTÉ, Antoine. «Martin Blais, L'autre Thomas d’Aquin, Montréal, Boréal, 1990 ». Vol. 20, n² 2 , p. 506-508.

DAIGLE, Christine. "Mathieu Kessler, L'esthétique de Nietzsche, Paris, PUF, 1998 ». Vol. 27, $\mathrm{n}^{\circ} 1$, p. 211-215.

DEROME, Germain. "Gérard Naddaf, L'origine et l'évolution du concept grec de phusis, Lewinston, New York : Queenston, Ontario, The Edwin Mellen Press, 1992 ». Vol. 23, $\mathrm{n}^{\circ}$ 1, p. 174-177.

DESJARDINS, Lucie. «Bernard Lamy, La rhétorique ou l'art de parler, éd. critique établie par Benoît Timmermans, Paris, PUF, $1998 »$. Vol. 27, n 1, p. 216-219.

DESMEULES, Louis. "Alfred Schmidt, Le concept de nature chez Marx, Paris, PUF, 1994 ». Vol. 24, n 1, p. 198-200.

DESPLAND, Michel. "Gérard Cholvy et Nadine-Josette Chaline (dir.), L'enseignement catholique en France aux XIX et XX ${ }^{e}$ siècles, Paris, Cerf, 1995 ». Vol. 24, nº 1, p. 198.

. "Patrice Vermeren, Victor Cousin. Le jeu de la philosophie et de l'État, Paris, L'Harmattan, $1995 »$. Vol. 24, n 1, p. 197.

- "Jose A. Prades, Persistance et métamorphose du sacré. Actualiser Durkheim et repenser la modernité, préface de Fernand Dumont, Paris, PUF, $1987 »$. Vol. 16, n 1, p. 212-218.

DESROCHES, Daniel. «Paul Ricœur, Éthique et responsabilité, textes réunis par J.-Ch. Aeschlimann, Neuchâtel, la Baconnière, 1994 ». Vol. 26, n 1, p. 155-157.

DESROCHES, Dominic. "H. J. Gensler, Questions d'éthique. Une approche raisonnée de quelques perspectives contemporaines, trad. de M.-C. Désorcy, Montréal, Chenelière/ Mc Graw-Hill, $2002 »$. Vol. 30, n² 2, p. 485-487.

. «David Brezis, Kierkegaard et les figures de la paternité, Paris, Cerf, 1999 ». Vol. 29, nº 1, p. $163-165$.

. "Jacques Caron, Kierkegaard aujourd'hui : Actes du Colloque de la Sorbonne, Odense, Odense University Press, 1998 ». Vol. 25, n² 2, p. 285-288.

DORION, Louis-André. «Olivier Reboul, Introduction à la rhétorique, Paris, PUF, 1991 » Vol. 20, n² 2, p. 508-511. 
. «B. Cassin (dir.), Positions de la sophistique, Paris, Vrin, 1986 ». Vol. 19, n 1, p. 134-139. . «Y. Lafrance, L. Paquet et M. Roussel, Les Présocratiques : bibliographie analytique (18791980), Montréal : Bellarmin; Paris : Les Belles-Lettres, 1988 ». Vol. 17, n 1, p. 151-154.

DOYON, Frédérique. «François Leroux, Figures de la souveraineté, Nietzsche et la question politique, Montréal, Hurtubise, $1997 »$. Vol. 24, n² 2, p. 431-435.

DROUIN, Sébastien. "Robert Challe, Difficultés sur la religion proposées au Père Malebranche, d'après le manuscrit de la Staatsbibliothek de Munich, édition de Frédéric Deloffre et François Moureau, Genève, Droz, 2000 ». Vol. 28, n 1, p. 226-228.

DUBOIS, Christian. "Sir Robert Filmer, Patriarcha ou le pouvoir naturel des rois, suivi des Observations sur Hobbes, trad. par Michaël Biziou, Colas Duflo, Hélène Pharabod, Patrick Thierry (dir.) et Béatrice Trotignon, Paris, L'Harmattan, 1991 ». Vol. 20, n² 2, p. 511-512.

DUCHESNEAU, François. "Renée Bouveresse, Leibniz, Paris, PUF, 1994 ». Vol. 22, n 1, p. $165-168$.

DUFOUR, Mario. "Gilles Deleuze et Félix Guattari, Qu'est-ce que la philosophie?, Paris, Éd. de Minuit, $1991 »$. Vol. 23, n 1, p. 177-181.

. «Jean Grondin, Kant. Avant/après, Paris, Critérion, 1990 ». Vol. 19, n 1, p. 141-144.

DUFOUR, Michel. "Donald Martel, L'anthropologie d'Althusser, Ottawa, Éd. de l'Université d'Ottawa, $1984 »$. Vol. 12, n² 2, p. 459-462.

DUFOUR, Mylène. "Jean-Louis Vieillard-Baron (éd.), De saint Thomas à Hegel, Paris, PUF, $1994 »$. Vol. 24, n 1, p. 187-191.

. "Philip M. Merklinger, Philosophy, Theology and Hegel's Berlin Philosophy of Religion, 1821-1827, Albany, University of New York Press, $1993 »$. Vol. 23, n² 2, p. 446-448.

. «Alfredo Ferrarin, Hegel interprete di Aristotele, ETS Editrice Pisa, 1990 ». Vol. 21, $\mathrm{n}^{\circ} 2$, p. 617-620.

DUFOUR, Richard. «Platon, Lachès - Euthyphron, intro. et trad. par Louis-André Dorion, Paris, Flammarion, $1997 »$. Vol. 25, n 1, p. 132-136.

DUGRÉ, François. "Bertrand Dumoulin, Recherches sur le premier Aristote (Eudème, De la philosophie, Protreptique), Paris, Vrin, $1981 »$. Vol. 16, n 1, p. 199-201.

DUHAMEL, André. "Monique Canto-Sperber, L'inquiétude morale et la vie humaine, Paris, PUF, $2001 »$. Vol. 29 , n 2, p. 388-391.

. "Robert Spaemann, Bonheur et bienveillance. Essai sur l'éthique, traduit par Stéphane Robillard, Paris, PUF, $1997 »$. Vol. 26, n 1, p. 159-163.

DUMAS, Denis. "Alexis Philonenko, Le transcendantal et la pensée moderne. Études d'histoire de la philosophie, Paris, PUF, 1990 ». Vol. 17, n² 2, p. 203-209.

DUMOUCHEL, Daniel. «David Pugh, Dialectic of Love : Platonism in Schiller's Æsthetics, Montréal : McGill; Kingston : Queen's University Press, 1996 ». Vol. 25, n 2, p. 283-285.

. "Manfred Frank, Jean-Paul Larthomas et Alexis Philonenko, Sur la Troisième Critique, Combas, Éd. de l’Éclat, 1994 ». Vol. 23, n 1, p. 181-183.

. «Marc Sherringham, Introduction à la philosophie esthétique, Paris, Payot, 1992 ». Vol. 22, n² 2, p. 505-508.

- "Jean-Marie Schaeffer, L'art de l'âge moderne. L'esthétique et la philosophie de l'art du XVIII siècle à nos jours, Paris, Gallimard, 1992 ». Vol. 21, n² 2, p. 620-624. 
. «Louis Dumont, Homo Æqualis II : L'idéologie allemande. France-Allemagne et retour, Paris, Gallimard, $1991 »$. Vol. 21, n 1, p. 259-262.

DUMOUCHEL, Paul. «Thomas Nagel, Égalité et partialité, trad. par C. Beauvillard, Paris, PUF, 1994 ». Vol. 23, n 1, p. 183-185.

EGYED, Bela. «Jean Roy, Hobbes et Freud, Halifax, Dalhousie University Press, 1976 ». Vol. 8, $n^{\circ} 1$, p. 202-204.

FACENDIS, Dario De. «Barbara Cassin, L'effet sophistique, Paris, Gallimard, 1995 ». Vol. 25, $\mathrm{n}^{\circ} 1$, p. 121-125.

FAUCHER, Luc. «D. Andler (dir.), Introduction aux sciences cognitives, Paris, Folio, 1992 » Vol. 21, n 1, p. 262-267.

. "Daniel Dennett, La stratégie de l'interprète : le sens commun et l'univers quotidien, trad. de P. Engel, Paris, Gallimard». Vol. 19, n 1, p. 162-168.

FEDI, Laurent. «Patrice Vermeren, Le rêve démocratique de la philosophie d'une rive à l'autre de l'Atlantique, suivi de Amédée Jacques, Essai de philosophie populaire. Postface de Arturo Andres Roig, Paris, L'Harmattan, 2002 ». Vol. 29, n 2, p. 395-396.

FERLAND, Madeleine. «Paul Henry Thiry d'Holbach, Le Système de la Nature, ou Des lois du monde physique et du monde moral, Fayard, 1990 ». Vol. 19, n 1, p. 145-146.

FISETTE, Denis. "Jacques Derrida, Le problème de la genèse dans la philosophie de Husserl, Paris, PUF, $1990 »$. Vol. 18, n² 2, p. 184-188.

. «Edmund Husserl, La philosophie comme science rigoureuse, Paris, PUF, 1989». Vol. 17, $\mathrm{n}^{\circ} 2$, p. 222-225.

FOISY, Suzanne. «Louis Jacob (dir.), L’art et la norme, Montréal, Société 15/16, 1996 ». Vol. 25, $\mathrm{n}^{\circ} 2$, p. 300-304.

. «J. G. Fichte et F. W. J. Schelling, Correspondance (1794-1802), présentation, traduction et notes de Myriam Bienenstock, Paris, PUF, $1991 »$. Vol. 20, n 1, p. 202-203.

. «Mikel Dufrenne, L'oil et l’oreille, Essai, L'Hexagone, Montréal, 1987 ». Vol. 15, n 1, p. 234-240.

FOREST, Dominic. "Arturo Sangalli, Éloge du flou. Aux frontières des mathématiques et de l'intelligence artificielle, Montréal, Les Presses de l'Université de Montréal, 2001 ». Vol. 31, $\mathrm{n}^{\circ} 2$, p. 444-446.

FOURNIER, François. "Michel Robert (dir.), Fondements et étapes de la recherche scientifique en psychologie, Montréal : Chenelière et Stanké; Paris : Maloine S. A., 1982 ». Vol. 12, $\mathrm{n}^{\circ} 1$, p. 221-224.

FRANCESCHI, Paul. «John Leslie, Infinite Minds, Oxford, Oxford University Press, 2001 . Vol. 30, n 2, p. 453-455.

FRÉCHETTE, Guillaume. «Edmund Husserl, La représentation vide suivi de Les Recherches logiques, une cuvre de percée sous la direction de Jocelyn Benoist et Jean-François Courtine, Paris, PUF, $2003 »$. Vol. 31, n 1, p. 262-266

. «Jocelyn Benoist, Robert Brisart et Jacques English, Liminaires phénoménologiques : recherches sur le développement de la théorie de la signification de Husserl, Bruxelles, Publications des Facultés universitaires Saint-Louis, 1998 ». Vol. 27, n 1, p. 219-225.

GAGNON, Claude. "André Vidricaire (et collaborateurs), Figures de la philosophie québécoise après les troubles de 1837, Montréal, Université du Québec à Montréal, Département de philosophie, $1988 »$. Vol. 16, n² 2, p. 450-455. 
. "Guy-H. Allard (et collaborateurs), Jean Scot, écrivain: Actes du Ive colloque international de la SPES, Montréal : Bellarmin; Paris : Vrin, 1986 ». Vol. 14, n 1, p. 203-207.

. «Hilaire de Poitiers, La Trinité, trad. fr. de M ${ }^{\text {gr }}$ Albertus Martin, Paris, Desclée de Brouwer, $1981 »$. Vol. 10, n 2, p. 409-411.

. "Benoît Lemaire, L'espérance sans illusions. L'espérance chrétienne dans la perspective de Gustave Thibon, Montréal, Éditions Paulines, 1980 ». Vol. 9, n² 2, p. 337-340.

. «E. L. Fortin, Dissidence et philosophie au moyen-âge. Dante et ses antécédents, Montréal : Bellarmin; Paris : Vrin, Cahiers d'études médiévales de l'Institut d'études médiévales de l’Université de Montréal, 1981 ». Vol. 9, n 1, p. 175-178.

. «Ernest Joós (dir.), Scolastique, certitude et recherche; en hommage à Louis-Marie Régis, Montréal, Bellarmin, $1980 »$. Vol. 8, n 1, p. 199-202.

GAGNON, Martin. "Jean-Luc Marion, Étant donné. Essai d'une phénoménologie de la donation, Paris, PUF, Épiméthée, 1997 ». Vol. 25, n 1, p. 136-139.

. «Jeanne Delhomme, La pensée interrogative, Paris, PUF, 1993 ». Vol. 22, n 1, p. 169-173.

. «Maurice Rainville, L'expérience et l'expression. Essai sur la pensée de Merleau-Ponty, Montréal, Bellarmin, 1988 ». Vol. 16, n² 2, p. 410-418.

. «André Morazain et Salvatore Pucella, Éthique et politique. Des valeurs personnelles à l'engagement social, Montréal, Éd. du Renouveau pédagogique, 1988 ". Vol. 16, nº 1, p. 206-208.

GAGNON, Maurice. «Daniel Andler, Anne Fagot-Largeault et Bertrand Saint-Cernin, Philosophie des sciences I et II, Paris, Gallimard, 2002 ». Vol. 31, nº 1, p. 275-287.

. "Jacques J. Ruelland, Bibliographie des œuvres de Gaston Bachelard, ainsi que des divers ouvrages que sa pensée et sa personne ont inspirés, Montréal, Université du Québec à Montréal, Département de philosophie, 1980 ». Vol. 8, n² 2, p. 355-356.

GAGNON, Nicole. "Yvon Desrosiers (dir.), Religion et culture au Québec. Figures contemporaines du sacré, Montréal, Fides, $1986 »$. Vol. 14, n² 2, p. 444-450.

GAUTHIER, Yvon. "Michael Friedman, Dynamics of Reason, Stanford, CSLI Publications, $2001 »$. Vol. 31, n 1, p. 269-271.

. "Margaret Morrison, Unifying Scientific Theories. Physical Concepts and Mathematical Structures, Cambridge, Cambridge University Press, 2000 ». Vol. 30, n 1, p. 263-266.

. "Mark Steiner, The Applicability of Mathematics as a Philosophical Problem, Cambridge, Cambridge University Press, 1998 ». Vol. 30, n 1, p. 266-267.

. «Pierre Cassou-Noguès, Hilbert, Paris, Les Belles Lettres, 2001 ». Vol. 29, n 2, p. 391-392. . «André Stanguennec, Hegel. Une philosophie de la raison vivante, Paris, Vrin, 1998 ». Vol. 28, n 1, p. 232-233.

. «Jean-Pierre Belna, Cantor, Paris, Les Belles Lettres, 2000 ». Vol. 28, n 1, p. 238-239.

- . "Roland Omnès, Philosophie de la science contemporaine, Paris, Gallimard, Folio Essais, $1994 »$. Vol. 27, n² 2, p. 460-462.

. «Jean-Pierre Ginisti, La logique combinatoire, Paris, PUF, 1997 ». Vol. 26, n² 2, p. 375-376.

. "Jean-Pierre Belna, La notion de nombre chez Dedekind, Cantor, Frege, Paris, Vrin, $1996 »$. Vol. 25, n 1, p. 126-127.

. "Marco Panza et Jean-Michel Salanskis (dir. publ.), L'objectivité mathématique. Platonisme et structures formelles, Paris, Masson, 1995 ». Vol. 24, n 1, p. 217-220. 
. «Michel Paty, Einstein philosophe, Paris, PUF, 1993 ». Vol. 23, n 1, p. 185-187.

. "Jean Largeault, L'intuitionnisme, Paris, PUF, 1992 ». Vol. 21, n 1, p. 267-268.

. «Jean-Louis Gardies, Le raisonnement par l'absurde, Paris, PUF, 1991 ». Vol. 20, n² 2 , p. 513-514.

. «Gilles Gaston-Granger, La vérification, Paris, Odile Jacob, 1992 ». Vol. 20, n² 2, p. 514-516.

. «Alain Badiou, Le nombre et les nombres, Paris, Seuil, 1990 ». Vol. 20, n 1, p. 204-205.

. "Luc Brisson et F. Walter Meyerstein, Inventer l'univers. Le problème de la connaissance et les modèles cosmologiques, Paris, Les Belles Lettres, 1991 ». Vol. 19, n 1, p. 150-155.

. "Jean Andouze, Paul Musset et Michel Paty (dir.), Les particules et l'univers, Paris, PUF, $1990 »$. Vol. 18, n² 2, p. 197-198.

. «François Rivenc, Introduction à la logique, préf. de Jacques Bouveresse, Paris, Inédit, Petite Bibliothèque Payot, 198 ». Vol. 17, n 1, p. 165-166.

. «Jean-Baptiste Scherrer, Le théorème de Gödel, Paris, Seuil, 1989 ». Vol. 17, n 1, p. $157-159$.

. "Cahiers de l'École des sciences philosophiques et religieuses, 2-1987, publiés par les Facultés universitaires Saint-Louis, J. Deleval, Bruxelles, 1987 ». Vol. 16, n 1, p. 205-206.

GERVAIS, Richard. «Alain Martineau, Herbert Marcuse's Utopia, Montréal, Harveset House, $1986 »$. Vol. $15, n^{\circ} 1$, p. 240-243.

GIRARD René O. «Raymond Klibanski et David Pears (dir.), La philosophie en Europe, Paris, Gallimard/Unesco, 1993 ». Vol. 23, n 1, p. 188-190.

GIROUX, Claude. «Julien Naud, Une philosophie de l'imagination, Montréal : Bellarmin; Paris : Desclée, $1979 »$. Vol. 9, n² 2, p. 340-341.

. «Grapotte, Sophie : François Duchesneau, Guy Lafrance, et Claude Piché (dir.), Kant Actuel. Hommage à Pierre Laberge, Montréal : Bellarmin; Paris : Vrin, 2000 ». Vol. 30, $\mathrm{n}^{\circ} 2$, p. 466-470.

GRATTON, Claude. "Jacques Pierre, Mircea Éliade : le jour et la nuit. Entre la littérature et la science, Montréal, Hurtubise, 1989 ». Vol. 20, nº 1, p. 205-206.

GRAVEL, Pierre. «Leo Strauss et Joseph Cropsey, Histoire de la philosophie politique, trad. de O. Sedeyn, Paris, PUF, 1994 » et «Leo Strauss, Qu'est-ce que la philosophie politique?, trad. de O. Sedeyn, Paris, PUF, $1992 »$. Vol. 24, n² 2, p. 435-436.

. «René Girard, Shakespeare : les feux de l'envie, trad. de Bernard Vincent, Paris, Grasset, $1990 »$. Vol. 20, n 2, p. 516-518.

. "Hugo Ott, Martin Heidegger : Éléments pour une biographie, trad. de l'all. par J.-M. Belœil, Paris, Payot, $1990 »$. Vol. 19, nº 1, p. 146-150.

. «Étienne Souriau (dir.), Vocabulaire d'esthétique, Paris, PUF, 1990. Vol. 18, n² 2 , p. 188-189.

GUAY, Éric. «Bernard Bourgois, La philosophie allemande classique, Paris, PUF, 1995 ». Vol. 25, $n^{\circ} 1$, p. 129-132.

GUERTIN, Marie. "Catherine Larrère, L'invention de l'économie au XVIII siècle, Paris, PUF, $1992 »$. Vol. 21, n 1, p. 268-270.

HAMELIN, Guy. «Claude Panaccio, Le discours intérieur. De Platon à Guillaume d'Ockham, Paris, Seuil, $1999 »$. Vol. 29, n 1, p. 147-150. 
. «Hardy-Vallée, Benoit : Max Kistler, Causalité et lois de la nature, Paris, Vrin, 1999». Vol. 28, n², p. 447-450.

HÉBERT, Robert. «Éliane Gubin et Yvan Lamonde, Un Canadien français en Belgique au XIX ${ }^{e}$ siècle. Correspondance d'exil de L.-A. Dessaulles 1875-1878, Bruxelles, Palais des Académies, $1991 »$. Vol. 21, n 1, p. 271-272.

. «Mostafa Faghfoury (dir.), Analytical Philosophy of Religion in Canada, Ottawa, University of Ottawa Press, $1982 »$. Vol. 10, n² 2, p. 411-412.

. Collectif, Philosophie et littérature, Montréal, Bellarmin, 1979. Vol. 10, nº 1, p. 188-191. . «Stanley G. French (dir.), Philosophers Look at Canadian Confederation/La Confédération canadienne : qu'en pensent les philosophes? Montréal, Association canadienne de philosophie, 1979. Vol. 8, nº 1, p. 214-217.

HÉLAL, Georges. «Robert Lebeuf, Cosmic Presence, Montréal, Bellarmin, 1980 ». Vol. 9, n² 2, p. 359-361.

IMBEAULT, Marc. "Jean Grondin, L’horizon herméneutique de la pensée contemporaine, Paris, Vrin, 1993.»Vol. 23, n² 2, p. 454-456.

. "Jean Grondin, Einführung in die philosophische Hermeneutik, Darmstadt, Wissenschaftliche Buchgesellschaft, $1991 »$. Vol. 21, n 1, p. 272-273.

. «Henri Meschonnic, Le langage Heidegger, Paris, PUF, 1990 ». Vol. 18, nº 1, p. 182-184.

. «Paul Ricœur, Soi même comme un autre, Paris, Seuil, 1990 ». Vol. 18, n² 2, p. 190-191.

JANKELEVITCH, Sophie. "John Stuart Mill, Essais sur Tocqueville et la société américaine, trad. et prés. de P. Thierry, Paris, Vrin, 1994 ». Vol. 24, n 1, p. 191-193.

KATTAN, Emmanuel. «Blandine Banet-Kriegel, Les droits de l'homme et le droit naturel, Paris, PUF, $1989 »$. Vol. 17, n² 2, p. 214-217.

KAUFMANN, J. Nicolas. "Rudolf Bernet, La vie du sujet. Recherches sur l'interprétation de Husserl dans la phénoménologie, Paris, PUF, 1994 ». Vol. 24, n 1, p. 193-196.

. "Amartys Sen, Éthique et économique (et autres essais), trad. de l'anglais par Sophie Marnat, Paris, PUF, 1993 ». Vol. 22, n 1, p. 173-176.

. «Roberto Miguelez, Science, valeurs et rationalité, Ottawa, Éd. de l'Université d'Ottawa, $1984 »$. Vol. $12, n^{\circ} 1$, p. 225-229.

KNEE, Philip. «J. G. A. Pocok, Le moment machiavélien. La pensée politique florentine et la tradition républicaine atlantique, Paris, PUF, 1997 ». Vol. 25, n² 2, p. 281-283.

. "Jean Terrasse, De mentor à Orphée. Essais sur les écrits pédagogiques de Rousseau, Ville LaSalle, Hurtubise, $1992 »$. Vol. 21, n² 2, p. 624-627.

. "Albert Soboul, Contre nous de la tyrannie... Des relations idéologiques entre Lumières et Révolutions, Montréal, Hurtubise, 1990 ». Vol. 17, n² 2, p. 209-212.

LABELLE, Gilles. "Jacques Bidet, Théorie de la modernité. Suivi de : Marx et le marché, Paris, PUF, $1990 »$. Vol. 23, n² 2, p. 449-451.

LABERGE, Jean. «Marcil, Louise (dir.), La philosophie pour enfants. L'expérience de Lipman, Le Griffon d'argile, $1990 »$. Vol. 21, n 1, p. 274-278.

LACHAPELLE, Jean. «Richard Bett, Pyrrho. His Antecedents and his Legacy, Oxford, Oxford University Press, $2000 »$. Vol. 31, n 2, p. 452-457.

. "Yvon Gauthier, La philosophie des sciences. Une introduction critique, Montréal, Presses de l’Université de Montréal, 1995 ». Vol. 24, n 1, p. 220-222. 
LACROIX, André. "Maurice Gagnon et Daniel Hébert, 2000, En quête de science. Introduction à l'épistémologie, Montréal, Fides». Vol. 28, n 1, p. 236-237.

- "Alasdair MacIntyre, Quelle justice? Quelle rationalité?, traduction française de Michèle Vignaux d'Hollande, Paris, PUF, 1993. Vol. 23, n 1, p. 190-193.

. «Michel Rosier, L'État expérimentateur, Paris, PUF, 1993 ». Vol. 22, nº 1, p. 176-182.

LACROSSE, Joachim. "Jean-Marc Narbonne, La métaphysique de Plotin. Suivi de Henôsis et Ereignis: Remarques sur une interprétation heideggérienne de l'Un plotinien, deuxième édition revue et augmentée, Paris, Vrin, 2001. Vol. 31, nº 2, p. 450-452.

LAFLEUR, Gérald. "Alain Boyer (et autres), La pensée de Karl Popper et la science économique, numéro spécial de la revue Économie et Sociétés: Cahiers de l'Institut de sciences mathématiques et économiques appliquées publiées sous la dir. de M. François Perroux, tome 21, n 10, Paris, $1987 »$. Vol. 16, n 1, p. 218-230.

LAGUEUX, Maurice. "Dorval Brunelle, Socialisme, étatisme et démocratie, Montréal, Éd. Albert Saint-Martin, $1983 »$. Vol. 12, n 2, p. 454-459.

. "Normand Lacharité, Le modèle ER. Un système de catégories destiné à l’analyse des entreprises de recherche. Livre I, chapitre I : "Problématique, objectifs et stratégie du projet d'analyse". Montréal, Université du Québec à Montréal, Dép. de philosophie, $1981 »$. Vol. 9 , n² 2, p. 347-350.

LAMBERT, Pasquier. "Mathieu Kessler, Nietzsche ou le dépassement esthétique de la métaphysique, Paris, PUF, $1999 »$. Vol. 28, n 1, p. 228-232.

. «Roland Jaccard, L'enquête de Wittgenstein, Paris, PUF, 1998 ». Vol. 26, n² 2, p. 386-388.

. «Jean-Pierre Cometti, La maison de Wittgenstein, Paris, PUF, 1998 ». Vol. 26, n² 2, p. 388-391.

. "Antonia Soulez (dir.), Dictées de Wittgenstein à Wainsmann et pour Schlick, 2 vol., Paris, PUF, 1997. Vol. 25, n² 2, p. 296-300.

. «Jean-Pierre Cometti, Philosopher avec Wittgenstein, Paris, PUF, 1996 ». Vol. 25, n² p. 294-296.

LAMBERT, Roger. «Théodore Geraets (dir.), Hegel, l'Esprit absolu, Ottawa, Éd. de l'Université d'Ottawa, n² 26, $1984 »$. Vol. 14, n 1, p. 218-221.

. "John Burbidge, On Hegel's Logic, Fragments of a Commentary, Atlantic Highlands, N. J., Humanities Press, 1981 ». Vol. 10, n 1, p. 180-182.

LANGLOIS, Luc. «Dominique Folscheid, La philosophie allemande de Kant à Heidegger, Paris, PUF, $1993 »$. Vol. 22, n² 2, p. 545-547.

LARMORE, Charles. "Raymond Klibansky et Josiane Boulad-Ayoub (dir.), La pensée philosophique d'expression française au Canada, Québec, Presses de l'Université Laval, 1998 ». Vol. 27, nº 2, p. 456-460.

LARUE, Pierre. "Jocelyn Benoist et F. Merlini (dir.), Historicité et spatialité, le problème de l'espace dans la pensée contemporaine, Paris, Vrin, 2001 ». Vol. 30, n 1, p. 276-280.

LASVERGNAS, Isabelle. "Chantal Saint-Jarre, Du Sida, l'anticipation imaginaire de la mort et sa mise en discours, Paris, Denoël, 1994 ». Vol. 23, n² 2, p. 456-461.

LATRAVERSE, François. «Paul-Marcel Lemaire, Les signes, sauvages. Une philosophie de langage ordinaire, Ottawa, Éd. de l'Université d'Ottawa, 1980 ». Vol. 8, n 1, p. 209-211. 
LAURIER, Daniel. «François Latraverse, La pragmatique : histoire et critique, Bruxelles, Mardaga, $1987 »$. Vol. 16, n² 2, p. 446-450.

. "Luc Bégin et autres, Pragmatisme et pensée contemporaine. Université de Sherbrooke, Dép. de philosophie, Cahiers de philosophie, 1984 ». Vol. 12, n 1, p. 232-236.

LAVOIE, Jourdain. "Benoit Pruche, Existant et acte d'être. Essai de philosophie existentielle, tome 1, Introduction générale. Critique existentielle; tome 2, Analytique existentielle, Paris,Tournai : Desclée; Montréal : Bellarmin, 1977-1980». Vol. 9, n 1, p. 178-184.

LEBEUF, Maxime. "Gérard Raulet, Histoire et citoyenneté, Paris, PUF, 1996 ». Vol. 24, n², p. 437-439.

LEBLANC Martin. «Stéphane Chauvier, Justice internationale et solidarité, Nîmes, Éd. Jacqueline Chambon, $1999 »$. Vol. 30, n² 2, p. 458-461.

LECLERC, André. «Donald Peterson, Wittgenstein's Early Philosophy. Three Sides of the Mirror, Toronto/Buffalo, University of Toronto Press, $1990 »$. Vol. 18, n 1, p. 179-182.

LECLERCQ, Bruno. «Denis Fisette et Sandra Lapointe (dir.), Aux origines de la phénoménologie. Husserl et le contexte des Recherches Logiques, Paris : Vrin; Québec : Presses de l’Université Laval, 2003 ». Vol. 31, n 2, p. 439-443.

LEDUC, Christian. "Jean-Baptiste Rauzy, La doctrine leibnizienne de la vérité. Aspects logiques et ontologiques, Paris, Vrin, $2001 »$. Vol. 30, n² 2, p. 479-482.

LEGAULT, Georges A. «Gilbert Boss, Introduction aux techniques de la philosophie. Analyse de l'idée de justice, Zurich, Éditions du Grand Midi, 1989 ». Vol. 20, n 1, p. 206-207. . "Gilbert Hottois, Le paradigme bioéthique. Une éthique pour la technoscience, Bruxelles, ERPI Science, $1990 »$. Vol. 20, n 1, p. 208-209.

LEPAGE, François. "Michel J. Blais, La logique, une introduction, Montréal : Presses de l'Université de Montréal; Sherbrooke : Presses de l'Université de Sherbrooke, 1985 ". Vol. 13, nº 1, p. 188-194.

LEROUX, Georges. «Jean-François Pradeau (dir.), Platon : les formes intelligibles. Sur la forme intelligible et la participation dans les dialogues platoniciens, Paris, PUF, 2001 ». Vol. 31, $\mathrm{n}^{\circ} 1$, p. 251-254.

. «Plotin, Traité 9 (VI 9), intro., trad., commentaire et notes par Pierre Hadot, Paris, Cerf, 1994. » Vol. 24, n 1, p. 185-187.

. "Frederic M. Schroeder, Form and Transformation. A Study in the Philosophy of Plotinus, Montréal : McGill; Kingston : Queens University Press, 1992 ». Vol. 23, n² 2, p. 439-440.

"Richard Bodéüs, Aristote et la théologie des vivants immortels, Montréal : Bellarmin; Paris : Les Belles-Lettres, $1992 »$. Vol. 22, n² 2, p. 509-517.

. "Thomas DeKoninck et Guy Planty-Bonjour (dir.), La question de Dieu selon Aristote et Hegel, Paris, PUF, 1991 ». Vol. 22, nº 1, p. 182-185.

. «Richard Rorty, L’homme spéculaire, trad. de l'anglais par Thierry Marchaisse, Paris, Seuil, $1990 »$. Vol. 20, n 1, p. 209-212.

. "Jean-François Mattei (dir.), La naissance de la raison en Grèce : Actes du Congrès de Nice, mai 1987, Paris, PUF, $1990 »$. Vol. 19, n 1, p. 131-134.

- "Michel Despland, The Education of Desire : Plato and the Philosophy of Religion, Toronto, University of Toronto Press, $1985 »$. Vol. 14, n 1, p. 213-218. 
- "Georges A. Legault et Luc Bégin, Le Québec face à la formation morale, Sherbrooke, Service à l'édition et à la recherche de la Faculté des arts de l'Université de Sherbrooke, Cahiers de philosophie, 1983; Anita Caron (et collaborateurs), Les parents et le statut confessionnel de l'école au Québec, Québec, Presses de l'Université du Québec, 1984 ». Vol. 13, n² 2, p. 403-408.

. "A. H. Armstrong, L'architecture de l'univers intelligible dans la philosophie de Plotin. Une étude analytique et historique, trad. de l'anglais par Josiane Boulad-Ayoub et Danièle Letocha, Ottawa, Éd. de l'Université d'Ottawa, 1984 ». Vol. 13, nº 1, p. 179-182.

. «Lloyd P. Gerson (ed.), Graceful Reason. Essays in Ancient and Medieval Philosophy Presented to Joseph Owens, CSSR, on the Occasion of his Seventy-Fifth Birthday and Fiftieth Anniversary of his Ordination, Toronto, Pontifical Institute of Medieval Studies, $1983 »$. Vol. 12, n 1, p. 229-231.

. "Pierre Gravel et Timothy Reiss (dir. publ.), Tragique et tragédie dans la tradition occidentale/Tragedy and the Tragic in Western Culture, Montréal, Déterminations, 1983 ». Vol. 11, n 2, p. 425-427.

. "Aristote, Éthique à Eudème, trad. par Vianney Décarie, Montréal : Presses de l’Université de Montréal; Paris : Vrin, 1978 ». Vol. 10, nº 1, p. 177-180.

. «Luc Brisson, Platon 1958-1975. Lustrum 20 (1977), Göttingen, Vandenhoeck \& Ruprecht, $1979 »$. Vol. 8, n 1, p. 197-199.

LEROUX, Jean. «Robert Nadeau, Vocabulaire technique et analytique de l'épistémologie, Paris, PUF, $1999 »$. Vol. 28, n 1, p. 239-242.

. «Normand Lacharité, Un modèle informationnel de la représentation, Montréal, Dép. de philosophie de l'Université du Québec à Montréal, Cahiers Recherches et théories, $1987 »$. Vol. $15, \mathrm{n}^{\circ} 1$, p. 225-230.

LEROUX, Serge. "Jean-Jacques Rousseau, Émile ou de l'éducation, édition, introduction, bibliographie de Tanguy L'Aminot; texte, notes et index par François et Pierre Richard, Paris, Bordas, $1992 »$. Vol. 20, n 1, p. 213-222.

LÉTOURNEAU, Alain. "Maurice Blondel, Euvres complètes, tome $1: 1893$. Les deux thèses, texte présenté par Claude Troisfontaines, Paris, PUF, 1995 ». Vol. 24, n 1, p. 200-203.

LIGHT, Steve. «Carlo Michelstaedter, La persuasion et la rhétorique, Sergio Campailla (dir. publ.), trad. de l'italien par Marilène Raiola, Milan, Éd. de l'Éclat, 1989 ». Vol. 23, nº 1, p. 198-201.

LUGG, Andrew, et Donald MCDONELL. «Serge Robert, Les révolutions du savoir : théorie générale des ruptures épistémologiques, Longueuil, Le Préambule, 1979». Vol. 11, n²1, p. 203-205.

MARINEAU, René F. «Lucie Bonnette, Le fondement religieux de la pensée de Jung, Montréal, Fides, 1986. Vol. 14, n 2, p. 435-437.

MARION Mathieu. «Jérôme Dokic et Pascal Engel, Ramsey. Vérité et Succès, Paris, PUF, 2001. Vol. 31, n 1, p. 266-269.

. "Lucie Antoniol, Lire Ryle aujourd'hui. Aux sources de la philosophie analytique, De Boeck, Bruxelles, 1993. Vol. 22, n² 2, p. 518-522.

. "Gerhard Heinzmann (dir.), Poincaré, Russell, Zermelo et Peano. Textes de la discussion (1906-1912) sur les fondements des mathématiques: des antinomies à la prédicativité, Paris, Librairie scientifique et technique Albert Blanchard, 1986 ». Vol. 18, n 1, p. 184-188. 
MARQUIS, Jean-Pierre. «Angèle Kremer-Marietti, La philosophie cognitive, Paris, PUF, 1994 ». Vol. 23, n², p. 461-464.

MARTEL, Marie. "Gérard Genette, L'œuvre de l'art, immanence et transcendance, Paris, Seuil, 1994 ». Vol. 26, n 1, p. 163-167.

MARTIN, Axelle. "Josiane Boulad-Ayoub et François Blanchard, Les grandes figures du monde moderne, Paris, L'Harmattan, $2001 »$. Vol. 29, n² 2, p. 411-418.

MARTINEAU, Alain. "André Vachet, Marcuse : La révolution radicale et le nouveau socialisme. Essai de synthèse, Éd. de l'Université d'Ottawa, n 11, 1986 ». Vol. 14, n 2, p. 455.

MATHIEU, Olivier. "Jean-Luc Marion, De surcroît, Paris, PUF, 2001 ». Vol. 30, n 1, p. 280-285.

MAZILU, Daniel. «Jean-Marc Narbonne, Hénologie, ontologie et Ereignis (Plotin - Proclus Heidegger), Paris, Les Belles Lettres, $2001 »$. Vol. 29, n 1, p. 161-163.

MCDONELL, Donald, et Andrew LUGG. "Serge Robert, Les révolutions du savoir : théorie générale des ruptures épistémologiques, Longueuil, Le Préambule, 1979». Vol. 11, nº 1, p. 203-205.

MELKEVIK, Bjarne. «Institut de formation continue du Barreau de Paris (sous l'égide de), Le doute et le droit, Paris, Dalloz, 1994 ». Vol. 24, n² 2, p. 439-440.

. «William Baranès et Marie-Anne Frison-Roche, La justice. L'obligation impossible, Paris, Autrement, Série Morales, 1994. Vol. 24, nº 1, p. 208-209.

. «François Terré (dir.), Le suicide, Paris, PUF, 1994 ». Vol. 22, n 1, p. 185-187.

MERCIER, Benoit. "Yves Roy, Autorité politique et liberté, Montréal, Éditions VLB, 1988 ». Vol. 16, n² 2, p. 407-410.

MICHAUD, Ginette. "Catherine Malabou (dir.), “Derrida”, numéro spécial de la Revue philosophique de la France et de l'étranger, n 2, $1990 »$. Vol. 18, n² 2, p. 180-182.

MICHAUD, Pierre. "Georges Tavard, Les jardins de saint Augustin : Lecture des Confessions, Montréal, Montréal : Bellarmin; Paris : Cerf, 1988 ». Vol. 16, n 1, p. 210-212.

MIGUELEZ, Roberto. "Josiane Boulad-Ayoub, Mimes et parades. L'activité symbolique dans la vie sociale, Paris, L'Harmattan, 1995 ». Vol. 24, n 1, p. 209-213.

. "À la recherche du sens/In search of meaning, Ottawa, Revue de l'Université d'Ottawa/University of Ottawa, Quarterly, vol. 55, n 4 ». Vol. 18, n 1, p. 175-178.

MORAIS, Marceline. "Alain Boyer, Hors du temps. Un essai sur Kant, Paris, Vrin, 2001 » Vol. 29, n² 2, p. 392-394.

MOSER, Walter. «Peter Royle, The Sartre-Camus Controversy. A Literary and Philosophical Critique, Ottawa, University of Ottawa Press, 1982 ». Vol. 13, n 1, p. 182-188.

NADEAU, Christian. "Thomas Hurka, Virtue, Vice and Value, Oxford, Oxford University Press, $2001 »$. Vol. 29, n² 2, p. 383-388.

. "Miguel E. Vatter, Between Form and Event : Machiavelli's Theory of Political Freedom, Dordrecht, Kluwer, 2000 ». Vol. 29, n 1, p. 157-161.

. "Jean-Fabien Spitz, L'amour de l'égalité. Essai sur la critique de l'égalitarisme républicain en France 1770-1830, Paris, Vrin, 2000 ». Vol. 28, n² 2, p. 453-457.

. "Laurent Jaffro (coord.), Le sens moral. Une histoire de la philosophie morale de Locke à Kant, Paris, PUF, 2000 ». Vol. 28, n 2, p. 450-453.

. «Paolo Rossi, Les philosophes et les machines, 1400-1700, Paris, PUF, 1996 ». Vol. 25, $\mathrm{n}^{\circ} 2$, p. 304-306. 
. «Denis Kambouchner, L’homme des passions, Paris, Albin Michel, 1995 ». Vol. 24, n² 2, p. 440-442.

. «Christian Lazzeri, Force et justice dans la politique de Pascal, Paris, PUF, 1993 ». Vol. 23, n² 2, p. 441-443.

NADEAU, Robert. «François Duchesneau, La physiologie des lumières. Empirisme, modèles et théories, La Haye, Martinus Nijhoff, $1982 »$. Vol. 12, n 1, p. 217-221.

. "Guy H. Allard et Serge Lusignan (dir. publ.), Les arts mécaniques au moyen âge. Cahiers d'études médiévales, $n^{\circ}$ VII, Montréal : Bellarmin; Paris : Vrin, 1982 ». Vol. 11, $\mathrm{n}^{\circ} 1$, p. 206-209.

. «William H. Dray, Perspectives on History, London, Routledge \& Kegan Paul, Boston, Henley, $1980 »$. Vol. 8, n² 2, p. 363-365.

NÉRON, Pierre-Yves. "Kok-Chor Tan, Toleration, Diversity and Global Justice, University Park, Pennsylvania State University Press, $2000 »$. Vol. 30, n 2, p. 475-479.

NGUYEN, Vinh de. "Luc Ferry, Le nouvel ordre écologique. L'arbre, l'animal et l'homme, Paris, Grasset, $1992 »$. Vol. 20, n² 2, p. 521-524.

ONG-VAN-CUNG, Kim Sang. «Hans Jonas, Le phénomène de la vie : vers une biologie philosophique, trad. de D. Lories, Bruxelles, De Boeck Université, 2001 ». Vol. 29, nº 1, p. 150-153.

PAGEAU Véronique. "Sophie de Grouchy, Lettres sur la sympathie, suivies de Lettres d'amour à Mailla Garat, textes revus, présentés et annotés par Jean-Paul de Lagrave, Montréal, Presses de l'Université du Québec, Cahiers de recherches et théories ". Vol. 21, n 1 , p. 279-281.

PANACCIO, Claude. "Claude Lafleur, Quatre introductions à la philosophie au XIII siècle. Textes critiques et étude historique, Montréal : Institut d'études médiévales; Paris : Vrin, 1988 ». Vol. 17, n² 2, p. 201-203.

PAQUIN, Louis-Claude. "Chiara Crisciani et Claude Gagnon, Alchimie et philosophie au moyen âge, Montréal, l'Aurore/Univers, 1980 ». Vol. 8, n² 2, p. 356-357.

PARADIS, André. "Josiane Boulad-Ayoub et autres, Les épiphanies idéologiques. Théorie, idéologie, société, Montréal, Dép. de philosophie de l'Université du Québec à Montréal, $1981 »$. Vol. 9 , n² 2, p. 362-363.

PATAUT, Fabrice. «Daniel Laurier (dir.), Essais sur le sens et la réalité, Montréal : Bellarmin; Paris : Vrin, $1991 »$. Vol. 22, n² 2, p. 523-532.

PELLAND, Ginette. "Pierre Gravel, Politiques, fermmes, pouvoir. Éléments d'une théorie de l’otage (Essai sur le théâtre de Jean Racine), Montréal, Éditions VLB». Vol. 19, n 1 , p. 139-141.

PELLERIN, René. «Gilles Lane, À quoi bon la philosophie? Longueuil, Le Préambule, 1982 ». Vol. 11, n 1, p. 213-216.

PICHÉ, Claude. "Jocelyn Benoist, Kant et les limites de la synthèse : le sujet sensible, Paris, PUF, $1996 »$. Vol. 24, n² 2, p. 442-444.

. "Béatrice Longuenesse, Kant et le pouvoir de juger. Sensibilité et discursivité dans l'analytique transcendantale de la Critique de la raison pure, Paris, PUF, 1993 ». Vol. 23, n², p. 443-446.

- "Jean-Luc Marion, Réduction et donation. Recherches sur Husserl, Heidegger et la phénoménologie, Paris, PUF, 1989 ». Vol. 20, n 1, p. 222-224.

. "Alain Bontat, Heidegger, Paris, PUF, 1989». Vol. 17, n² 2, p. 225-227. 
PINARD, Sylvain. "Marcel Hénaff, Claude Lévi-Strauss, Paris, Éd. Pierre Belfond, 1991 » Vol. 20, n 1, p. 224-228.

PLOURDE, Simonne. "Nicole Bonnet, Immanence et transcendance chez Teilhard de Chardin, préface d'André A. Devaux, Montréal : Bellarmin; Paris : Cerf, n 11, 1987 ». Vol. 15, $\mathrm{n}^{\circ} 2, \mathrm{p} .487-489$.

POAMÉ, Lazare M. "Gilbert Hottois, De la renaissance à la postmodernité. Une histoire de la philosophie moderne et contemporaine, Bruxelles, De Boeck Université, 1997 ». Vol. 24, $\mathrm{n}^{\circ} 2$, p. 444-446.

PONCE, Véronica. "Marc Ereshesfky, The Poverty of the Linnaean Hierarchy : A Philosophical Study of Biological Taxonomy, Cambridge, Cambridge University Press, 2001 ». Vol. 31, $\mathrm{n}^{\circ} 1$, p. 271-275.

PRAT, Sébastien. «Philip, Knee, La parole incertaine : Montaigne en dialogue, Québec, Presses de l'Université Laval, 2003 ». Vol. 31, n 2, p. 446-450.

QUIVIGER, Pascale. "Christian Saint-Germain, Écrire sur la nuit blanche : l'éthique du livre chez Emmanuel Lévinas et Edmond Jabès, Montréal, Presses du l'Université du Québec, $1992 »$. Vol. 21, n 2, p. 627-631.

RANGER, Philippe. "Josiane Boulad-Ayoub, Vers une redéfinition matérialiste du concept de culture, Montréal, Dép. de philosophie de l’Université du Québec à Montréal, Presses de l’Université du Québec, 1987 ». Vol. 16, n² 2, p. 426-431.

. «Machiavel, Le Prince, trad. et présentation de Gérard Allard, Sainte-Foy, Le Griffon d'argile, $1984 »$. Vol. 16, n² 2, p. 422-426.

. «Normand Lacharité, Un modèle informationnel de la représentation : contribution aux théories du symbolique, Montréal, Dép. de philosophie de l'Université du Québec à Montréal, Presses de l’Université du Québec, 1987 ». Vol. 16, n² 2, p. 431-436.

. «Jean-Paul Desbiens, L'actuel et l'actualité, Sainte-Foy, Le Griffon d'Argile, 1986 ». Vol. 14, n² 2, p. 451-454.

RATTÉ, Michel. «Bruce Bégout, La généalogie de la logique : Husserl, l'antéprédicatif et le catégorial, Paris, Vrin, $2000 »$. Vol. 30, n² 2, p. 449-453

RENAULT, Marc. "Jean Theau, Certitudes et questions de la raison philosophique, Ottawa, Éd. de l'Université d'Ottawa, 1985 ». Vol. 13, n² 2, p. 414-422.

RIOUX, Bertrand. «Johannès B. Lotz, Martin Heidegger et Thomas d'Aquin, trad. de l'all. par Philibert Secretan, Paris, PUF, $1988 »$. Vol. 16, n 2, p. 418-422.

RIOUX-BEAULNE, Mitia. «Marc Crépon, Le malin génie des langues. Nietzsche, Heidegger, Rosenzweig, Paris, Vrin, $2000 »$. Vol. 28, n² 2, p. 457-461.

ROBERT, Serge. "Jean Leroux, La sémantique dans les sciences physiques, Ottawa, Presses de l’Université d'Ottawa, 1988 ». Vol. 16, n 1, p. 230-234.

. "Georges Hélal, La philosophie comme pan-physique. La philosophie des sciences de A. N. Whitehead, Montréal, Éd. Bellarmin, 1979». Vol. 10, n 1, p. 186-188.

ROSE, Aimé-Pierre. «M. Gagnon et G. Legault (dir.), Philosophie et éducation, Cahiers de l'Association canadienne-française pour l'avancement des sciences, n 19, 1984 ». Vol. 13, $n^{\circ} 2$, p. 408-414.

ROSS, Don. "Michel Seymour, Pensée, langage et communauté. Une perspective anti-individualiste, Montréal : Bellarmin; Paris : Vrin». Vol. 24, n 1, p. 213-217. 
- "Serge Robert, Les mécanismes de la découverte scientifique, Ottawa, Presses universitaires d'Ottawa, 1993 ». Vol. 23, n 1, p. 204-210.

ROY, Manuel. "Christian Klotz, Selbstbewu? tsein und praktische Identität. Eine Untersuchung über Fichtes Wissenschaftslehre nova methodo, Philosophische Abhandlungen (vol. 84), Francfort-sur-le-Main, Vittorio Klostermann, 2002 ». Vol. 30, n 2, p. 470-473.

. "Jean-François Goubet, Fichte et la philosophie transcendantale comme science. Étude sur la naissance de la première Doctrine de la Science (1793-1796), Paris, L'Harmattan, 2002 ». Vol. 30, n², p. 473-475.

. «F. W. J. Schelling, Exposition de mon système de la philosophie et Sur le vrai concept de la philosophie de la nature, suivi de J. G. Fichte, Sur l'exposition du système de l'identité de Schelling, trad. et prés. par E. Cattin, Paris, Vrin, 2000 ». Vol. 29, n 1, p. 155-157. . "Jean-Christophe Goddard, La philosophie fichtéenne de la vie : le transcendantal et le pathologique, Paris, Vrin, 1999». Vol. 29, n 1, p. 153-155.

. "Jean-Christophe Goddard (coord.), Fichte : le moi et la liberté, Paris, PUF, 2000 ». Vol. 28, n² 2, p. 461-466.

. «Violetta L. Waibel, Hölderlin und Fichte 1794-1800, Paderborn, Ferdinand Schöningh, $2000 »$. Vol. $28, n^{\circ}$ 1, p. 233-235.

ROY, Yves. "Gilles Lane, Pouvoir, justice et non-mépris, Montréal, Éditions VLB, 1989 ». Vol. 16, n 2, p. 405-407.

RUELLAND, Jacques G. "Claude Paris, La liberté. Libre volonté et maîtrise de soi, Québec, Éd. C.G., $1988 »$. Vol. 17, n² 2, p. 227-228.

. «Claude Paris, Le discours religieux. Une réflexion, Québec, Éd. C.G., 1988 ». Vol. 17, $\mathrm{n}^{\circ} 2$, p. 228-229.

. «Lizette Jalbert et Lucille Beaudry (dir.), Les métamorphoses de la pensée libérale, sur le néo-libéralisme actuel, Québec, Presses de l’Université du Québec, 1987 ». Vol. 17, $\mathrm{n}^{\circ} 1, \mathrm{p} .162-163$.

. «Louise Poissant, Machinations, Montréal, Société d'Esthétique du Québec, 1989». Vol. 17, nº 1, p. 161-162.

. "Luc Chartrand, Raymond Duchesne et Yves Gingras, Histoire des sciences au Québec, Montréal, Boréal, 1987 ». Vol. 15, n 2, p. 483-484.

. "Guy Lafrance (dir.), Gaston Bachelard. Profils épistémologiques, Ottawa, Presses de l'Université d'Ottawa, 1987 ». Vol. 15, n 2, p. 484-486.

. «Michel Germain, L'intelligence artificieuse, Montréal, L'Hexagone, 1986 ». Vol. 14, $\mathrm{n}^{\circ} 2$, p. $442-444$.

. «Danielle Blondeau (dir.), De l'éthique à la bioéthique : repères en soins infirmiers, Chicoutimi, Éd. Gaëtan Morin, 1986 ». Vol. 14, n² 2, p. 440-442.

. "Gérard Raulet, Humanisation de la nature, naturalisation de l'homme. Ernst Bloch ou le projet d'une autre rationalité, Paris, Klincksieck, 1982 ». Vol. 11, n 1, p. 210-212.

. La petite revue de philosophie, vol. 8, n 1, (aut. 1986)». Vol. 14, n² 2, p. 450-451.

SAINT-ARNAUD, Jocelyne. "Avortement Oui/Non, propos recueillis par Michel Burviana, Québec, Humanitas, $1988 »$. Vol. 17, n² 2, p. 229-232.

SAUVÉ, Denis. «Brian McGuiness, Wittgenstein. Les années de jeunesse : 1889-1921, trad. de Yvonne Tenenbaum, Paris, Seuil, $1991 »$. Vol. 20, n² 2, p. 518-519. 
SEYMOUR, Michel. «Donald Davidson, Actions et événements, trad. de l'américain par Pascal Engel, Paris, PUF, 1993 ». "Donald Davidson, Enquêtes sur la vérité et l'interprétation, trad. de l'américain par Pascal Engel, Nîmes, Éd. Jacqueline Chambon, 1993 ». Vol. 22, n² 2, p. 532-540.

SHEARSON, A. "Jean Grondin, Kant et le problème de la philosophie : l'a priori, Paris, Vrin, $1989 »$. Vol. 22, n 2, p. 540-544.

SIMARD, Jean-Claude. "Yvan Lamonde et Claude Lorin, Louis-Joseph Papineau. Un demisiècle de combats. Choix de textes et présentation, Montréal, Fidès, 1998 ". Vol. 29, n 1, p. 167-170.

. "Yves Gingras, Peter Keating et Camille Limoges, Du scribe au savant. Les porteurs du savoir de l'Antiquité à la révolution industrielle, Montréal, Boréal, 1999 ». Vol. 28, n² 2, p. 466-469.

. "Laurent-Michel Vacher, Découvrons la philosophie avec François Hertel, Montréal, Liber, 1995. Vol. 26, n 2, p. 383-386.

. «En collaboration, Le phénomène IXE-13, Québec, Presses de l'Université Laval, 1984. Vol. 14, n 1, p. 208-213.

. "Alexis Klimov, Éloge de l’homme inutile, Québec, Éd. du Beffroi, 1983; Diversions. Huit opérations poétiques pour une stratégie métaphysique, Québec, Éd. du Beffroi, 1983; Veilleurs de nuit, Québec, Éd. du Beffroi, 1984 ». Vol. 12, n² 2, p. 445-451.

SIMARD, Yvon. «G. Bouchard, L. Giroux et G. Leclerc, L'utopie aujourd'hui, Les Presses de l'Université de Montréal/Éd. de l’Université de Sherbrooke, 1985 ». Vol. 13, n² 2, p. $422-429$.

SOSOE, Lukas K. «Hannah Arendt, Juger. Sur la philosophie politique de Kant, suivi de deux essais interprétatifs par Ronald Beiner et Myriam Revault d'Allonnes, Paris, Seuil, 1991 ». Vol. 22, n 1, p. 187-190.

. "Guy Bourgeault, L'éthique et le droit face aux nouvelles technologies biomédicales, Montréal, Presses de l’Université de Montréal, 1990 ». Vol. 21, n 1, p. 282-289.

SOUSA Melo, Candida JACI DE. «Josette Lanteigne, La question du jugement? Paris, L'Harmattan, $1993 »$. Vol. 23, n 1, p. 193-196.

STROUD, Sarah. «Ruwen Ogien (dir.), Le réalisme moral, Paris, PUF, 1999». Vol. 28, n 1 , p. 219-223.

TALIN, Christian. "Lygia Négrier-Dormont et Stamatios Tzitzis, Criminologie de l'acte et philosophie pénale. De l'ontologie criminelle des Anciens à la victimologie appliquée des Modernes, préface de H.-A. Schwartz-Liebermann von Wahlendorf, postface de George C. Christie, Paris, Litec, 1994 ». Vol. 24, n 1, p. 203-206.

STAMATIOS Tzitzis, La philosophie pénale, Paris, PUF, 1996 ». Vol. 24, n 1, p. 206-208.

TAVAGLIONE, Nicolas. «Bertrand, Russell, Le pouvoir, trad. de l'anglais par Michel Parmentier, Québec, Les Presses de l'Université Laval et les Éditions Syllepse, 2003 ». Vol. 31, nº 2, p. 436-439.

TCHAO, Joseph. "Charles Murin, Nietzsche problème, généalogie d'une pensée, Montréal, Presses de l'Université de Montréal, 1979 ». Vol. 8, n² 2, p. 352-355.

TEYSSEIRE, Daniel. "Jacques Piquemal, Essais et leçons d'histoire de la médecine et de la biologie, préf. de Georges Canguilhem, Paris, PUF, 1993 ». Vol. 23, n 1, p. 201-204.

THÉRIAULT, Mélissa. "Roger Pouivet, L'œuvre d'art à l'âge de sa mondialisation. Un essai d'ontologie de l'art de masse, Bruxelles, La Lettre volée, 2003 ». Vol. 31, n 1, p. 258-261. 


\section{$500 \cdot$ Philosophiques / Automne 2004}

TOURNIER, François. "Gilbert Hottois, Penser la logique. Une introduction technique, théorique et philosophique à la logique formelle, Bruxelles, De Boeck-Wesmael, 1990 ». Vol. 21, n² 2, p. 631-636.

. "Jacques G. Ruelland, De l'épistémologie à la politique. La philosophie de l'histoire de K. R. Popper, Paris, PUF, 1991 ». Vol. 21, n² 2, p. 636-640.

. «Jacques Croteau, L’homme : sujet ou objet? Prolégomènes philosophiques à une psychologie scientifico-humaniste, Montréal : Bellarmin; Paris : Desclée, 1981 ». Vol. 9, n² 2, p. 351-354.

. "Guy Massicotte, L'histoire problème. La méthode de Lucien Febvre, St-Hyacinthe : Edisem; Paris : Maloine, $1981 »$. Vol. 8, n² 2, p. 360-362.

TREMBLAY, Robert. «Nicole Everaert-Desmedt, Le processus interprétatif. Introduction à la sémiotique de Ch. S. Peirce, Liège, Pierre Mardaga éditeur, 1990 ». Vol. 18, n² 2, p. 195-197.

TURGEON, Marc. «Pierre Macherey, À quoi pense la littérature? Exercices de philosophie littéraire, Paris, PUF, $1990 »$. Vol. 18, n 2, p. 182-183.

. «Guy Bouchard, Le procès de la métaphore, Montréal, Hurtubise, 1984 ». Vol. 12, n 1, p. 236-241.

. "Jacques Derrida, L'oreille de l'autre (otobiographies, transferts, traductions), textes et débats avec Jacques Derrida, sous la dir. de Claude Lévesque et Christie V. McDonald, Montréal, Éditions VLB, 1982 ». Vol. 10, n 1, p. 182-186.

. «Marc Chabot, Chroniques masculines, Québec, Éditions Pantoute, 1981 ». Vol. 9, $n^{\circ} 2$, p. 344-347.

TURMEL, Patrick. «Engin F. Isin, Being Political. Genealogies of Citizenship, Minneapolis, University of Minnesota Press, 2002 ». Vol. 30, n 1, p. 285-289.

VALEVIIUS, Andrius. "Alban Urbanas, La notion d'accident chez Aristote, Montréal, Bellarmin, 1988 ». Vol. 17, n 1, p. 159-160.

VALLÉE, Richard. «Pascal Engel, Lire Davidson. Interprétation et holisme, Paris, l'Éclat, 1994 ». Vol. 23, n 2, p. 464-467.

. «Steven Davis (dir.), Pragmatics. A reader, New York/Oxford, Oxford University Press, 1991. Vol. 19, n 1, p. 157-162.

VEILLETTE, Claude. "Claude Piché, Kant et ses épigones. Le jugement critique en appel, Paris, Vrin, $1995 »$. Vol. 26, n², p. 392-395.

VIDRICAIRE, André. "Émile Chrétien, Le Québec philosophique, Montréal, McGraw-Hill, $1991 »$. Vol. 20, n 2, p. 519-521.

. «P. Wyczynski, F. Gallays et S. Simard (dir.), L'essai et la prose d'idées au Québec, t. VI, publié par le Centre de recherche en civilisation canadienne-française de l'Université d'Ottawa, Montréal, Fides, 1985 ». Vol. 15, n 1, p. 230-234.

. "Jean-Louis Allard, L'éducation à la liberté, ou la philosophie de l'éducation de Jacques Maritain, Ottawa : Éd. de l'Université d'Ottawa; Grenoble : Presses de l'Université de Grenoble, 1978 ». Vol. 8, n² 1, p. 206-208.

VIGNEAULT, Luc. «Julia Kristeva, Le génie féminin : Hannah Arendt, Paris, Fayard, 1999 ». Vol. 30, n² 2, p. 461-465.

VOLANT, Éric. "René Pellerin, Théories et pratiques de la désaliénation, Montréal, L'Hexagone, $1983 »$. Vol. 13, n 1, p. 195-198. 
WEINSTOCK, Daniel Marc. "Otfried Höffe, Principes du droit, trad. de Jean-Christophe Merle, Paris, Cerf, $1993 »$. Vol. 23, n 1, p. 196-198.

. «Jocelyne Couture (dir.), Éthique et rationalité, Liège, Éditions Pierre Mardaga, 1992 ». Vol. 22, n 1, p. 190-194.

- "Allen Buchanan, Secession: The Morality of Political Divorce from Fort Sumter to Lithuania and Quebec, Boulder, Westview Press, 1991». Vol. 20, n 1, p. 228-231.

. "Bernard Williams, L'éthique et les limites de la philosophie, trad. de Marie-Anne Lescourret, Paris, Gallimard, 1990 ». Vol. 20, n 1, p. 232235.

ZANINETTI, Andrea. "Christian Lazzeri, Spinoza, puissance et impuissance de la raison, Paris, PUF, $1999 »$. Vol. 28, n² 2, p. 469-471.

\section{Interventions}

ARMOUR, Leslie. "Religion et philosophie au Québec et au Canada anglais ». Vol. 9, $\mathrm{n}^{\circ}$ 2, p. 307-316.

ATLAN, Henri. "Rationalité scientifique et rationalité du mythe». Vol. 22, n² 2, p. 265-280. BÉDARD, Michel. «Philosophie et culture générale». Vol. 5, n² 2, p. 381-396.

BOUET, Jacques. «La définition de la simultanéité pour des corps en mouvements rectilignes et pour ceux en mouvements circulaires ». Vol. 24, n 2, p. 341-344.

BOULAD-AYOUB, Josiane. «De David et Jean-Jacques à Jean et à Jacques. Un échange inconnu entre Hume et Rousseau ». Vol. 23, n 1, p. 151-161.

. «Présentation». Présentation de l'intervention «Interdictions philosophiques». Vol. 5, $\mathrm{n}^{\circ} 1$, p. $179-180$

BRAYBROOKE, David. "À la recherche d'une justice de structure au Nouveau-Brunswick». Vol. 3, n 1, p. 123-129.

BRODEU, Jean-Paul. «Se taire, dit-il ?». Vol. 6, n 1, p. 201-207. . «Sur les justifications de la philosophie institutionnalisée». Vol. 3, n 1, p. 109-122.

BUNGE, Mario. «Culture et inculture». Vol. 13, n² 2, p. 347-351.

CAUCHY, Venant. «Entretien avec Monsieur Venant Cauchy». Entretien mené par Émile Chrétien. Vol. 17, n 1, p. 127-141.

CHABOT, Marc. "Roger Savoie, Le philosophe chat ou les ruses du désir, Montréal, Quinze/Prose exacte, $1980 »$. Vol. 8, n 1, p. 185-196.

CHAMPAGNE, René. "Texte à plusieurs voix autour d'un livre ou les suites d'une table ronde sur Le singulier de Marc Renault ». Vol. 10, n 1, p. 127-151.

CHANDLER, Tertius. "À propos de Moïse : deux notes de recherche». Vol. 22, n² 2, p. 297-299.

CHARETTE, Léon. «Texte à plusieurs voix autour d'un livre ou les suites d'une table ronde sur Le singulier de Marc Renault ». Vol. 10, n 1, p. 127-151.

. «L'idéologie dans l'éducation». Vol. 3, n 2, p. 289-297.

CHRÉTIEN, Émile. «Entretien avec Monsieur Venant Cauchy». Vol. 17, n 1, p. 127-141. 
COLLIN, Claude. "Critique d'une critique». Réponse au compte rendu critique de Louise Marcil-Lacoste : "L'expérience philosophique ». Vol. 8, n 1, p. 149-166.

CÔTÉ, Marcel, et Gilles PARADIS. «Les dictionnaires généraux de philosophie en langue française ». Vol. 23, n² 2, p. 341-358.

COUTURE, Jocelyne. "À quoi servent les faits normatifs ? ". À propos de «L'autonomie de la morale» de Charles Larmore. Vol. 24, $\mathrm{n}^{\circ}$ 2, p. 329-335.

COUTURIER, Fernand. «Le pays de la culture ». Vol. 5, n¹, p. 195-199.

D’HONDT, Jacques. «Utopie et liberté ». Vol. 1, n² 2, p. 141-156.

DAOUST, Jean-Guy. «Endoctrinement et politisation dans l'enseignement de la philosophie ou d'un faux problème». Vol. $3, \mathrm{n}^{\circ}$ 1, p. 94-101.

DESJARDINS, Pierre. «Les loteries nationales : une exploitation malheureuse? » Vol. 12, ${ }^{\circ} 1$, p. 191-196.

DREVET, Patrick. «Le parvis du temple». Vol. 22, n 1, p. 95-102. . «La parole risquée». Vol. 22, n 1, p. 103-115. . «Le désir de dire». Vol. 21, n² 2, p. 549-561.

DUCHARME, Léonard. «Morale objective et loi naturelle». Vol. 4, n 1, p. 102-109.

DUCHESNEAU, François. Discours prononcé à l'occasion de la remise du prix André Laurendeau 1992. Vol. 21, n 1, p. 191-195.

. «Figures de l'empirisme». Discours de réception à la Société royale du Canada. Précédé d'une courte présentation par Josiane Boulad-Ayoub. Vol. 12, n 2, p. 411-420.

EYNDE, Laurent Van. «De l'Action painting à la mort de l'art : à propos de Jackson Pollock ». Vol. 22, nº 2, p. 281-296.

FORSTER, Jean-Paul. «Avec les yeux d'un étranger : Les lettres d'un Persan de George Lyttleton ». Vol. 23, n 1, p. 139-149.

GAUTHIER Yvon. «Réplique à Pierre Raymond». Vol. 5, n¹, p. 192-194.

GODIN, Christian. «L'encyclopédisme hors d'Europe». Vol. 23, n² 2, p. 359-369.

GODIN, Guy. «Pour un bilan culturel du Nouveau Monde». Vol. 11, n 1, p. 185-190.

GUÉRIN, Michel. «Le concept topoḯtique». Vol. 24, n 1, p. 127-140.

HOUDE, Roland. «Genre et tendances. L'essai : sous-ensemble d'un ensemble». Vol. 10, n² p. 403-407.

. «La référence n'est pas à l'index (saint Thomas aujourd'hui)». Vol. 6, n 2, p. 341-346.

IMLAY, Robert. «Texte à plusieurs voix autour d'un livre ou les suites d'une table ronde sur Le singulier de Marc Renault». Vol. 10, nº 1, p. 127-151.

JANICAUD, Dominique. «Le temps de la nature et la mesure du temps». Vol. 23, n 2, p. 327-339.

JOOS, Ernest. «Le fondement de la morale et l'enseignement de la morale». Vol. 4, $\mathrm{n}^{\circ} 1$, p. $117-125$.

KERWIN, Larkin. "Les contributions de la science à la philosophie au XXe siècle ». Vol. 16, $\mathrm{n}^{\circ}$ 1, p. 149-161.

KLIBANSKY, Raymond. "L'université allemande dans les années trente : notes autobiographiques». Vol. 18, n² 2, p. 139-157.

LARMORE, Charles. «L'autonomie de la morale». Vol. 24, n² 2, p. 313-328. 
LECOURT, Dominique. «Les nouvelles philosophies de la nature». Vol. 20, n 1, p. 159-176.

LEGAULT, Georges A. «L'université et la crise “comptable” ». Vol. 9, n² 2, p. 317-336. . «La rhétorique des discours sur l'indépendance : l'argumentation historique». Vol. 7, $\mathrm{n}^{\circ} 1$, p. 101-130.

. «Législation et droits de l’homme». Vol. 2, n² 2, p. 342-354.

LEROUX, Georges. "Modernité des Grecs. L'importance de la pensée grecque et les raisons de l'enseigner dans le programme collégial de philosophie». Vol. 24, n 1, p. 141-160.

. «Expérience, communauté, institution. Réflexion sur la spécialisation de l'éducation morale au primaire ". Vol. 14, n² 2, p. 413-423.

. «La recherche et le comité de déontologie à l'université». Vol. 5, nº1, p. 200-208.

LIPMAN, Matthew. «Entrevue avec M. Matthew Lipman». Vol. 12, n² 2, p. 393-409.

MARCIL-LACOSTE, Louise. «Du sens commun à l'égalité ». Discours de réception à la Société royale du Canada. Vol. 15, n² 2, p. 453-464.

. «Le monopole confessionnaliste dans son rapport avec sa réflexion sur l'éthique.» Vol. 4, $\mathrm{n}^{\circ} 1$, p. 125-136.

. «Quand l’État devient philosophe». Vol. 2, n², p. 354-363.

MARGOLIS, Joseph. «Pour réhabiliter la notion d'incorporation des entités culturelles : réponse à Dale Jacquette». Vol. 13, n² 2, p. 333-343.

MEUNIER, Jean-Guy. "Le livre blanc de "la politique québécoise du développement culturel". Esquisse critique d'une philosophie de la culture». Vol. 6, n 2, p. 347-360.

MORIN, Serge J. «Sur l'injustice de structure ». Vol. 1, n 1, p. 171-192.

NADEAU, Robert. "Contre le scientisme. Pour l'ouverture d'un nouveau front ». Vol. 13, n², p. 353-368.

. «Hommage à Hugues Leblanc, philosophe logicien ». Vol. 13, n 1, p. 131-145.

. «Présentation». Présentation de l'intervention «L'enseignement de la morale et la question de ses fondements». Vol. 4, n 1, p. 101.

. «L'enseignement de la morale dans une perspective relativiste». Vol. 4, nº 1, p. 109-117.

. «Présentation». Présentation de l'intervention «La philosophie institutionnelle : critique et/ou idéologie?». Vol. 3, nº 1, p. 93.

. «Présentation». Présentation de la série d'interventions «Autour de la loi 50 ». Vol. 2, $\mathrm{n}^{\circ} 2$, p. 341-342.

NIELSEN, Kai. «La longue marche à travers les institutions : de Wittgenstein à Marx». Vol. 13, $\mathrm{n}^{\circ} 1$, p. 113-129.

NORCIA, Vincent Di. "Le fédéralisme, l'État, et la démocratie ». Vol. 8, n 1, p. 167-184.

O’NEAL, John. "Le continuum corps-esprit et l'économie de notre être selon Bonnet ». Vol. 19, $\mathrm{n}^{\circ} 1$, p. 87-111.

PANACCIO, Claude. «La responsabilité de la clarté». Vol. 14, n 1, p. 175-181.

. «Le projet de loi 50 et la place des droits de l'homme dans le système juridique ». Vol. 2, $\mathrm{n}^{\circ} 2$, p. 364-375.

PARADIS, Gilles. «La documentation en philosophie : bibliographies courantes ». Vol. 6, n 1 , p. 177-199. 
PARADIS, Gilles, et Marcel CÔTÉ. «Les dictionnaires généraux de philosophie en langue française ». Vol. 23, n² 2, p. 341-358.

PATRY, Marcel. «La fonction critique de l'université ». Vol. 2, n 1, p. 155-167.

PELLERIN, René. «Réflexion et responsabilités sociales». Vol. 5, n 2, p. 397-416.

PESTIEAU, Joseph. "Quel développement et pour qui ?». Vol. 16, n 2, p. 327-346.

PETIT, Jean-Claude. «La traduction française d'un ouvrage de H. G. Gadamer, Warheit und Methode». Vol. 10, n 1, p. 153-155.

POAMÉ, Lazare Marcelin. «Regard sur la philosophie de la technique en Allemagne ». Vol. 21, $\mathrm{n}^{\circ} 1$, p. $197-211$.

POULIN, Richard. «Savoir et croire. Sur le Pen et autres menus détails». Vol. 16, n 2, p. 359-371.

PROULX, Jean. «Par-delà l'idéologie et le soupçon : l'authenticité intellectuelle ». Vol. $3, \mathrm{n}^{\circ} 1$, p. 101-109.

PRUCHE, Benoît. «Discussion ». Contribution à l'intervention «L'enseignement de la morale et la question de ses fondements ». Vol. 4, n 1, p. 136-137.

RAULET, Gérard. «L'appel de l'histoire. La théorie critique de l'école de Francfort face au contexte français ». Vol. 9, n 1, p. 163-174.

RAYMOND, Pierre. «Les “Interdictions philosophiques” ». Vol. 5, n¹, p. 181-188. . «Réponse à Yvon Gauthier». Vol. 5, n¹, p. 189-191.

RENAULT, Marc. «Texte à plusieurs voix autour d'un livre ou les suites d'une table ronde sur Le singulier de Marc Renault». Vol. 10, n 1, p. 127-151.

SERRES, Michel. «L'anthropologie des sciences : un programme pour la philosophie?» Entrevue avec Michel Serres, 8 octobre 1986. Vol. 14, n 1, p. 147-171.

SOSOE, Lukas K. «Autonomie de la morale ou morale de l'autonomie». Vol. 24, n 2 , p. 337-339.

TALIN, Christian. "De l'infanticide en Chine au XVIII" siècle. Considérations sur les facteurs sociaux». Vol. 24, n 1, p. 91-125.

TEYSSEIRE, Daniel. «Le poète, le pédagogue et le républicain : Ginguené au service de l'institution publique». Vol. 22, n 1, p. 117-135.

VILLEMAIRE, Luc. «Paul Nizan, Les chiens de garde, Paris, François Maspero, 1932 ». Vol. 11, $\mathrm{n}^{\circ} 1$, p. $175-184$.

VOHO Sahi, A. «Entre figures de la philosophie : réflexion sur les Figures de la philosophie québécoise». Vol. 16, n² 2, p. 347-358.

\section{Tables rondes}

BOI, Luciano. «La géométrie : clef du réel ? Pensée de l'espace et philosophie des mathématiques». Vol. 24, n² 2, p. 389-430.

. "Leibniz sur l'espace, le continu et la substance : mathématique, physique et métaphysique ». Vol. 22, n 2, p. 407-436.

BOULAD-AYOUB, Josiane. "Les récurrences du platonisme chez Descartes ». Vol. 23, n 2 , p. 405-415. 
CHARBONNEAU, Louis. "Quelques réflexions sur Descartes et les mathématiques ». Vol. 22, $n^{\circ} 2$, p. 353-369.

DUCHESNEAU, François. «Le modèle du vivant dans la physique cartésienne ». Table ronde sur «Descartes : le quatrième centenaire». Vol. 23, n 2, p. 389-404.

. «La dynamique de Leibniz entre mathématiques et métaphysique. Réplique à Yves Gingras et à Luciano Boi». Vol. 22, n² 2, p. 437-463.

DUMOUCHEL, Daniel. «Descartes : discours et méthode». Vol. 23, n² 2, p. 373-387.

GAGNON, Maurice. «Métaphysique, théorie scientifique et expérience chez Descartes : ambiguïtés et difficultés». Vol. 22, n² 2, p. 371-383.

GAUTHIER, Yvon. «Commentaire de A Model of the Universe de Storrs McCall». Vol. 22, n 2, p. $481-487$.

GINGRAS, Yves. «La dynamique de Leibniz : métaphysique et substantialisme ». Vol. 22, n², p. 395-405.

GOMBAY, André. «Nous entrerons dans la carrière ». Vol. 22, n 2, p. 345-351.

MACHILDON, Louis. «Le modèle de l'univers de Storrs McCall». Vol. 22, n 2, p. 473-480.

MCCALL, Storrs. "Réponse à Yvon Gauthier, Louis Machildon et Serge Robert». Vol. 22, n² 2, p. 489-503.

MICHEL, Alain. «Espace et intelligibilité mathématiques : sur le problème mathématique de l'espace ». Vol. 24, n² 2, p. 349-366.

MOYAL, Georges J. D. «L'hypothèse du rêve et les vérités de la raison ». Vol. 23, n² 2, p. 417-425.

NADEAU, Robert. «Présentation». Table ronde sur «Le problème mathématique de l'espace». Vol. 24, n² 2, p. 345-347.

. «La philosophie des sciences au Québec : introduction». Table ronde sur «La philosophie des sciences au Québec». Vol. 22, n², p. 337-341.

PETITOT, Jean. "Philosophie transcendantale et objectivité physique». Vol. 24, n² 2, p. 367-388.

ROBERT, Serge. "Le dualisme ontologique de Storrs McCall». Vol. 22, n² 2, p. 467-472.

SHEA, William. «En guise de réponse à mes amis». Vol. 22, n 2, p. 385-392.

\section{Bulletins}

CLOUTIER, Yvan. Sartriana québécoise : chronologie, bibliographie et médiagraphie commentées. Vol. 16, n² 2, p. 373-393.

GARCEAU, Benoît. La philosophie analytique de la religion : contribution canadienne (19701975). Vol. 2, n² 2, p. 301-339.

GIROUX, Laurent. Colloque sur "La philosophie de l'histoire et la pratique historienne aujourd'hui ", à l'Université d'Ottawa, du 18 an 20 avril 1980. Compte rendu du colloque. Vol. 7, n² 2, p. 383-402.

HÉBERT, Robert. Introduction à l'histoire du concept de réflexion : position d'une recherche et matériaux bibliographiques. Vol. 2, n 1, p. 131-153.

KING-FARLOW, John. L'immutabilité $d u$ Dieu biblique et $d u$ Deus Thomisticus. Réponse à Benoît Garceau. Vol. 3, n² 2, p. 285-288. 


\section{Philosophiques / Automne 2004}

LABERGE, Pierre. Dix années d'études canadokantiennes (1968-1978). Vol. 5, n² 2, p. 331-380.

LAFRANCE, Yvon. Les études platoniciennes : contribution canadienne (1970-1977). Vol. 4, $\mathrm{n}^{\circ} 1$, p. 51-99.

LAMONDE, Yvan. L'histoire de la philosophie au Canada français (de 1920 à nos jours) : Sources et thèmes de recherche. Vol. 6, $\mathrm{n}^{\circ}$ 2, p. 327-339.

LANDRY, Albert M. La pensée philosophique médiévale : contribution canadienne (1960-1973). Vol. 1, n² 2, p. 111-139.

LATRAVERSE, François. Les études wittgensteiniennes au Canada : état de la recherche, 19701984. Vol. 12, n 1, p. 197-209.

MARCIL-LACOSTE, Louise. L'essai en philosophie : problématique pour l'établissement d'un corpus. Vol. 13, n 1 , p. 65-111.

MCKINNON, Alastair. Les études kierkegaardiennes au Canada. Vol. 9, n 1, p. 147-161.

PLOURDE, Simonne. Présence de la pensée de Gabriel Marcel au Canada (1940-1978). Vol. 6, $\mathrm{n}^{\circ} 1$, p. 147-173.

Frédéric Tremblay

Université du Québec à Montréal 